\title{
Structural and functional characterization of oligomeric states of proteins in RecFOR pathway
}

Santosh Kumar Chaudhary ${ }^{\mathrm{a}}$, Mohanapriya Elayappan ${ }^{\mathrm{b}}$, Jeyaraman Jeyakanthan ${ }^{\mathrm{c}}$ and Kanagaraj Sekar $^{\text {b* }}$

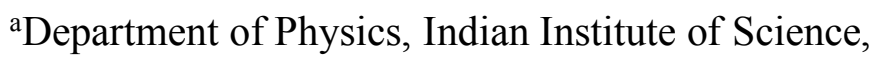
Bangalore 560012, India

${ }^{b}$ Department of Computational and Data Sciences, Indian Institute of Science, Bangalore 560012, India

and

cDepartment of Bioinformatics, Alagappa University, Karaikudi 630003, India

*Corresponding author

E-mail : sekar@iisc.ac.in

Phone : 91-80-22933059 / 22933060 / 23600551

Fax : 91-80-23600085 


\section{$\underline{\text { Abstract }}$}

RecFOR pathway is the principal repair pathway for double strand break and single strand gap repair in Thermus thermophilus. RecF and RecR exist as monomer and dimer in solution, interestingly; they undergo condition-dependent dimerization and tetramerization, respectively during the DNA break repair. However, their importance in protein-protein and protein-DNA interactions remains elusive. In this study, the three-dimensional crystal structures of the wild type RecF and RecR proteins are determined. Thereafter, the structural information is used to mutate the interface residues to cysteine to stabilize the dimeric and tetrameric states of the RecF and RecR proteins, respectively. A comparative study for their interactions with other cognate proteins and ssDNA in native and SSB (single strand binding protein) bound states was performed. RecF or RecFR complex displays a negligible affinity towards ssDNA. Conversely, the RecF mutants and its complexes with wild type RecR showed affinity towards ssDNA, suggesting, distinct modes of interaction of RecF and RecFR complex for ssDNA binding. In the presence of RecO, the stabilized tetrameric RecR showed a lower binding affinity for ssDNA as compared to the SSB bound ssDNA, indicating the importance of tetrameric RecR in stabilizing the RecOR complex on the SSB coated ssDNA. This provides an insight into the reduction of the binding affinity of SSB proteins with the ssDNA, which in turn enhances the recruitment of RecA for strand exchange.

\section{Keywords}

Dimeric TtRecF; Tetrameric TtRecR; Binding affinity; Heterohexamer; RecOR complex; RecFR complex

\section{Abbreviations}

SSB, single strand binding; $\mathrm{K}_{\mathrm{D}}$, Binding Affinity; ssDNA, single strand DNA; dsDNA, double strand DNA 


\section{Introduction}

The RecFOR pathway proteins play an important role in the restart of stalled replication fork and homologous recombination in prokaryotes (Bidnenko et al., 1999). RecBCD and RecFOR are the two major repair pathways present in prokaryotes. The RecBCD pathway is mainly responsible for the double strand DNA (dsDNA) break repair, whereas, the RecFOR pathway is required for single strand DNA (ssDNA) gap repair. However, in the absence of RecBCD pathway proteins, the RecFOR pathway also participates in dsDNA break repair. In contrast to other prokaryotes, the RecBCD pathway is absent and RecFOR is the principal DNA repair pathway in D. radiodurans, B. subtilis and T. thermophilus. In a DNA break event, the helicase RecQ unwinds the DNA (Harmon \& Kowalczykowski, 1998) and the RecJ protein creates a 3' overhang by 5'-3' exonuclease activity (Lovett \& Kolodner, 1989). The 3' overhang of DNA is subsequently coated with the SSB (single strand binding) proteins. The RecF, RecO and RecR proteins facilitate the loading of the RecA protein on SSB coated 3' overhang (Morimatsu \& Kowalczykowski, 2003). The RecA protein removes SSB proteins from the 3' tail of the DNA and forms a nucleoprotein filament. The nucleoprotein filament mediates heteroduplex formation between the ssDNA and the complementary homologous dsDNA for homologous recombination (Shibata et al., 1979; Handa et al., 2009; McEntee et al., 1979).

The RecR protein from the RecFOR pathway is reported as an essential repair mediator protein for all the homologous recombination and DNA repair pathways in M. tuberculosis (Gupta et al., 2015). RecR exists as a dimer in solution; however, the tetrameric assembly of RecR is reported in the crystal structures from D. radiodurans and T. tengcongensis (Tang et al., 2012; Lee et al., 2004). The tetrameric assembly of RecR monomers generates a central cavity, large enough to pass a dsDNA. The tetrameric assembly of RecR is required for the interaction with RecF and RecO proteins (Honda et al., 2008; Timmins et al., 2007). The proteins, RecF and RecO interact with the RecR in the ratio 4:2 (4RecR:2RecO/2RecF) (Tang et al., 2012; Timmins et al., 2007). $\mathrm{RecF}$ and RecO proteins exist as a monomer in solution. The RecF protein shows ATP dependent dimerization and dimer dependent ATPase activity upon DNA binding (Koroleva et al., 2007). The monomeric RecF protein does not show any ATPase activity and is active only in the presence of DNA (Tang et al., 2018). A recent study on RecF from T. tengcongensis revealed that the RecF forms a dimer in the presence of ATP, whereas in the presence of non-hydrolysable ATP analogue 
$(\mathrm{ATP} \gamma \mathrm{S})$, the dimeric interface changes and the helical region of DNA binding domain is involved in dimerization (Timmins et al., 2007).

RecFOR pathway proteins form various conditional homo and heteromeric complexes in solution. The roles of their oligomerization in protein-protein and protein-DNA interactions are not clear. In this study, the RecF and RecR proteins from $T$. thermophilus (TtRecF and TtRecR) are successfully crystallized and the high-resolution crystal structures of TtRecF and TtRecR proteins are solved. A dimeric interface of TtRecF is modeled based on the dimeric crystal structure of RecF from T. tengcongensis. To stabilize the dimeric state of TtRecF protein, cysteine mutations are performed on its interface residues. Similarly, the tetrameric TtRecR structure is also stabilized by di-sulfide bridges. The interaction properties of the cysteine mutants of RecF and RecR have been compared with the respective wild type proteins. The stabilized tetrameric RecR shows distinct binding properties with the ssDNA, when complexed with RecF and RecO proteins as compared to the wild type RecR. Wild type RecF shows negligible affinity towards ssDNA, interestingly, the mutant RecF shows affinity with the ssDNA and it increases in the presence of the wild type RecR. This suggests that RecF might have distinct binding modes for the ssDNA and dsDNA. Overall, the present study provides insights into the roles of the oligomeric states of RecF and RecR proteins in protein-protein and protein-DNA interactions. 


\section{Materials and methods}

\section{Mutagenesis, Protein expression and purification}

The $r e c \mathrm{~F}$, rec $\mathrm{O}$, rec $\mathrm{R}$ and $s s b$ genes from $T$. thermophilus cloned in $\mathrm{pET} 11$ a vector were brought from RIKEN, Japan. The mutants $\mathrm{TtRecR}_{\mathrm{G} 169 \mathrm{C}}, \mathrm{TtRecF}_{\mathrm{D} 57 \mathrm{C}}$ and $\mathrm{TtRecF}_{\mathrm{S} 261 \mathrm{C}}$ were generated using the inverse PCR technique. The primer details are provided in supplementary Table S1. The proteins were expressed in E. coli BL21DE3 (RIL) cells. These cells were harvested and suspended in lysis buffer. The lysis buffer used for TtRecR, TtRecR $\mathrm{G}_{169 \mathrm{C}}, \mathrm{TtRecO}$ and TtSSB consists of Tris$\mathrm{HCl} \mathrm{pH} 7.5,150 \mathrm{mM} \mathrm{NaCl}$ and 5mM $\beta$-merchaptoethanol, whereas RecF, RecF D57C $_{\text {and }} \mathrm{RecF}_{\mathrm{S} 261 \mathrm{C}}$ lysis buffer consists of Tris- $\mathrm{HCl} \mathrm{pH} 7.5,500 \mathrm{mM} \mathrm{KCl,} 25 \mathrm{mM}$ Sucrose, $5 \mathrm{mM}$ lysozyme and 5 mM $\beta$-merchaptoethanol. TtRecR, TtRecR ${ }_{\mathrm{G} 169 \mathrm{C}}$ and TtSSB were purified using anion exchange column and TtRecF, TtRecF ${ }_{\mathrm{D} 57 \mathrm{C}}, \mathrm{TtRecF}_{\mathrm{S} 261 \mathrm{C}}$ and $\mathrm{TtRecO}$ were purified using HiTrap Heparin HP column (GE healthcare). All the purified proteins were further polished using gel filtration column (Hi-load 16/600 Superdex200pg, GE healthcare) pre-equilibrated with the buffer containing 20mM HEPES pH 7.5 and $150 \mathrm{mM} \mathrm{NaCl}$ (Supplementary Fig. S1). The protein concentrations were measured using Bradford assay.

\section{Crystallization, Data Collection and processing}

The proteins TtRecF and TtRecO were successfully crystallized using under oil microbatch (greiner plate) technique. The drops contained $2 \mu 1$ of protein and $2 \mu 1$ of the crystallization condition. Silicon and paraffin oil $(3: 1)$ of $7 \mathrm{ml}$ was used in the plate. The crystallization condition of TtRecF consists of $0.1 \mathrm{M}$ Sodium malonate $\mathrm{pH}$ 7.0, 0.1M HEPES $\mathrm{pH} 7.0$ and $0.5 \% \mathrm{v} / \mathrm{v}$ Jeffamine ED-2001 pH 7.0 and TtRecR in 0.2M Potassium chloride, 0.05 M HEPES pH 7.5 and $35 \% \mathrm{v} / \mathrm{v}$ Pentaerythritol propoxylate. The data was collected at home source (Molecular Biophysics Unit, Indian Institute of Science, Bangalore, India) and BM14 beam line (European Synchrotron Radiation Facility (ESRF), Grenoble, France). The data sets were processed using iMOSFLM (Xu \& Freitas, 2009), xia2 (Holm \& Laakso, 2016), DENZO and SCALEPACK (Tang et al., 2018).

\section{Structure solution and refinement}

The three dimensional crystal structures of DrRecF (PDB id - 2O5V) and DrRecR (PDB id 1VDD) were used as starting models for molecular replacement of TtRecF and TtRecR, 
respectively, using the program PHASER (Moncalian et al., 2004). The sequence identities between DrRecF and TtRecF, and DrRecR and TtRecR were $40 \%$ and $50 \%$, respectively. The crystal lattice of the wild type TtRecF and TtRecR belong to the primitive orthorhombic space groups, P22 $2_{1}$ and I222, respectively, with one molecule in the asymmetric unit. The reciprocal space refinement was carried out using maximum likelihood method implemented in REFMAC program (Koroleva et al., 2007), integrated with CCP4 program suite (Lee et al., 2013; Baker et al., 2001), while real space refinement was performed using COOT (Lee et al., 2013). The model was submitted to PDB_REDO (Tang et al., 2018; Webb et al., 1999) for geometry optimization followed by cycles of refinement using REFMAC and COOT. A total of $5 \%$ of the reflections were set aside for R-free calculations.

\section{Native PAGE and Electro Mobility Shift Assay (EMSA)}

TtRecR $(10 \mu \mathrm{M}), \operatorname{TtRecO}(10,20,40 \mu \mathrm{M})$ and $\mathrm{TtRecF}(10,20,40 \mu \mathrm{M})$ were incubated in $25 \mu \mathrm{l}$ of $20 \mathrm{mM}$ HEPES ( $\mathrm{pH} 7.5$ ) and $150 \mathrm{mM} \mathrm{NaCl}$. After $10 \mathrm{~min}$ at $50^{\circ} \mathrm{C}$, the samples were analyzed by polyacrylamide gel electrophoresis (PAGE) using $8 \%$ gel under non-denaturing conditions. The proteins were visualized by Coomassie Brilliant Blue R-250 (CBB) staining. Fluorescein (FAM) labelled $\mathrm{dT}_{35}$ at the 5' and 3' of the oligonucleotide was used for the EMSA experiments.

$2 \mu \mathrm{M}$ of FAM labelled $\mathrm{dT}_{35}$ was used for the experiments. Similar buffer and protein concentrations were taken, as used for native-PAGE analysis. DNA was visualized by Bio-rad chemidoc using fluorescein filter.

\section{Bio-layer Interferometry}

ForteBio's Super Streptavidin biosensors were used for the immobilization $50 \mathrm{nM}$ of biotinylated ssDNA (Biotin-5'GCAATGAAGTACGCTTGCCAGCTGCAGTCATGATTG3') (Sultana \& Lee, 2015). The binding affinities of TtRecR, TtRecR ${ }_{\mathrm{G} 169 \mathrm{C}}, \mathrm{TtRecF}, \mathrm{TtRecF}_{\mathrm{D} 57 \mathrm{C}}, \mathrm{TtRecF}_{\mathrm{S} 261 \mathrm{C}}$, TtRecO and TtSSB to the 5' biotinylated ssDNA were measured by biolayer interferometry using an Octet RED96 instrument (ForteBio). The buffer used for the association and dissociation comprised of $20 \mathrm{mM}$ HEPES pH 7.5, $150 \mathrm{mM} \mathrm{NaCl}, 0.01 \%$ Tween 20 and $0.1 \%$ BSA. $2 \mathrm{M}$ $\mathrm{MgCl}_{2}$ was used for final regeneration. 


\section{$\underline{\text { Results and Discussion }}$}

\section{Sequence and structure analysis of TtRecF protein}

TtRecF is crystallized in orthorhombic space group $\left(\mathrm{P} 22_{1} 2_{1}\right)$ with one molecule in the asymmetric unit (Table 1). RecF exists as a monomer in solution (Koroleva et al., 2007). The RecF protein has two domains viz ATPase domain and DNA binding domain (Fig. 1a). The RecF protein share a strong homology with Rad50 and structural maintenance of chromosome (SMC) proteins found in eukaryotes and archaea. Both the proteins are involved in DNA repair activities (Rojowska et al., 2014; Lehmann, 2005). The ATPase domain shares a significant structural similarity with Lobe I subdomain of the Rad50 head domain and SMC proteins. ATPase domain consists of $\alpha$-helix sandwiched between parallel $\beta$-sheets from the top and anti-parallel $\beta$-sheets from the bottom (Fig. 1a). A similar pattern is present in the ATPase domain of both Rad50 and SMC proteins. The second, DNA binding domain of RecF is mostly $\alpha$-helical and shares similarity with the Lobe II domain of Rad50 head domain (Supplementary Fig. S2). The RecF protein consists of Walker A, Walker B, D-loop and ABC signature motifs (Fig. 1a). The Walker A motif is an ATP binding loop with the consensus sequence, GXXGXGKST. It interacts with $\alpha$ - and $\beta$-phosphates of di- and tri-nucleosides. The ABC signature motif is involved in sensing the $\gamma$-phosphate of tri-nucleosides (Moncalian et al., 2004). The Walker B motif consists of four aliphatic amino acids followed by two negatively charged residues. The negatively charged residue coordinates with the $\mathrm{Mg}^{2+}$ ion or polarizes the attacking water molecule (Hanson \& Whiteheart, 2005) and the D-loop consists of an invariant aspartic acid of unknown function. Structure based sequence alignment of RecF proteins shows that these motifs are conserved across the organisms (Fig. 2).

\section{Dimerization of TtRecF protein}

RecF shows ATP dependent dimerization and dimer dependent DNA binding (Koroleva et al., 2007). Recently, the dimeric structure of RecF from Thermoanaerobacter tengcongensis (Tte) has been reported in complex with ATP and ATP $\gamma$ S (PDB ids: $5 Z 68$ and 5Z69). The dimeric interface of TteRecF in both the complexes are very different and the dimeric state in complex with ATP is regarded as the biologically relevant complex (Tang et al., 2018). The ATP bound dimeric TteRecF was used to model the dimeric interface of TtRecF. The dimeric TtRecF model was further refined and energy minimized using Rosetta docking refinement server (Fig. 1b) (Lyskov \& Gray, 2008; Moretti et al., 2018). The dimeric interface area of TtRecF was higher than that of 
the TteRecF. No conserved hydrogen bonds were found in any of the two dimers. However, they commonly share regions such as, ABC signature and Walker A motifs present at their dimeric interface. The percentage contribution of polar and non-polar interactions for the stabilization of dimeric interface in both the proteins are similar (Supplementary Fig. S3a and Supplementary Table S2).

The dimeric state of RecF is the prerequisite for binding to the dsDNA or at the junction of dsDNAssDNA (Morimatsu \& Kowalczykowski, 2003). The RecF shows minimal ATPase activity and its dimeric state might get stabilized at high concentrations $(2 \mathrm{mM})$ of ATP (Michel-Marks et al., 2010; Koroleva et al., 2007). The slow ATPase activity may support RecF for longer binding time at the dsDNA and may help in scanning the dsDNA for the break site. Unsurprisingly, its role in DNA repair still remains elusive. One way to characterize its roles is by stabilizing its dimeric interface. Thus, the modeled dimeric TtRecF interface residues, Asp57 and Ser261 were selected for mutation to cysteine residues $\left(\mathrm{TtRecF}_{\mathrm{D} 57 \mathrm{C}}\right.$ and $\mathrm{TtRecF}_{\mathrm{S} 261 \mathrm{C}}$ ) (Fig 1b). The TtRecF mutants $\left(\mathrm{ttRecF}_{\mathrm{D} 57 \mathrm{C}}\right.$ and $\left.\mathrm{ttRecF}_{\mathrm{S} 261 \mathrm{C}}\right)$ were purified in soluble fraction. The wild type TtRecF exits as a monomer in solution and formed dimer in the presence of ATP (Koroleva et al., 2007; Tang et al., 2018). Thus, it was expected that the cysteine residues would come closer in the presence of ATP molecules and form a disulfide bond in an oxidative environment resulting in a stable dimeric TtRecF. To our surprise, the purification of both the TtRecF cysteine mutants (ttRecF $\mathrm{D} 57 \mathrm{C}$ and $\mathrm{ttRecF}_{\mathrm{S} 261 \mathrm{C}}$ ) resulted in a non-canonical dimeric, as well as, monomeric population in solution. The dimeric populations of the mutant proteins are reduced to monomeric state after treatment with a reducing agent (Fig. 3). As mentioned earlier, the TteRecF shows that the two monomers can exist in totally different orientations in the presence of non-hydrolysable ATP (Aravind et al., 1998). This suggests that the dimeric orientations of RecF might depend on its surrounding environment (Supplementary Fig. 3b). Most importantly, ABC ATPases and DrRecF showed ATP dependent dimerization at high ATP and protein concentrations (Leiros et al., 2005; Koroleva et al., 2007). In contrast, $\mathrm{TtRecF}_{\mathrm{D} 57 \mathrm{C}}$ and $\mathrm{TtRecF}_{\mathrm{S} 261 \mathrm{C}}$ mutants formed di-sulphide bridges in the absence of ATP. Several attempts to crystallize the mutants remain unsuccessful. Coupled enzyme and calorimetric assays (using malachite green) were performed on the wild type TtRecF and its mutants to monitor the changes in the ATPase activity. These experiments showed negligible ATPase activity for the wild type as well as mutant TtRecF proteins, both in the presence and 
absence of dsDNA/gap DNA (gDNA). A more sensitive ATPase assay (radioactivity assay) might be helpful in determining the changes in ATPase activity.

\section{Structural analysis of TtRecR protein}

RecR from Thermus thermophilus (TtRecR) was purified and crystallized in I222 space group. The RecR structure from D. radiodurans (DrRecR) (PDB -id: 1VDD) was used as a search model for Molecular Replacement (MR) calculations (Table 1). The model was successfully generated for TtRecR in I222 space group. The TtRecR protein solved in I222 space group consists of one molecule in the asymmetric unit (Fig. 4a). Interestingly, a tetrameric assembly of the RecR protein was generated by combining the other three crystal symmetry mates. The tetrameric assembly of monomeric subunits ensued to a ring shaped structure with a central cavity. The dimensions of the central cavity are 30 and $35 \AA$, large enough to pass a dsDNA (Fig. 4b).

The RecR protein consists of a Helix hairpin Helix (HhH) motif, a Cys4 zinc-finger motif, a Toprim domain and a Walker-B motif (Fig. 4a). These motifs are highly conserved across prokaryotes (Fig. 5). Helix hairpin Helix $(\mathrm{HhH})$ motif is present at the N-terminal of the protein. It has a consensus sequence, $h x x h x G h G x x x A x x h h$ (where, $h$ is any hydrophobic residue and $\mathrm{x}$ could be any amino acid). The motif comprises two $\alpha$-helices joined by a hairpin loop. HhH motifs are commonly found in many of the DNA replication and repair proteins, such as Endonuclease III of E. coli, human DNA polymerase $\beta$ and Adenine DNA glycosylase (Doherty et al., 1996; Thayer et al., 1995). In RecR proteins, the HhH motifs are domain swapped with the other adjacent molecule in the crystal to form a dimeric structure. The conserved Lys23, Arg27 and the loop containing Leu17, Pro18, Gly19 and Ile20 are projected towards the central cavity of the tetrameric TtRecR structure (Honda et al., 2008; Honda et al., 2006). These residues might play an important role in DNA binding. The mutation of Lys23 and Arg27 at equivalent positions in DrRecR resulted in reduced DNA binding (Lee et al., 2004).

TtRecR comprised of four conserved cysteine residues, which coordinate with the $\mathrm{Zn}^{2+}$ ion to form a Cys4 zinc finger motif (Fig. 4a). The zinc finger motif in proteins is found to be important for the protein-protein and protein-DNA interactions (Kusakabe et al., 1999). The zinc finger is formed by a combination of two antiparallel $\beta$-strands and a $\alpha$-helix. In DNA binding proteins, the $\alpha$-helix binds to the major groove of the DNA. However, the Cys4 zinc-finger motif in RecR plays an important role in maintaining its three-dimensional structure. The mutation of individual 
cysteine residues has an effect on the protein stability; however, its interaction with the DNA or RecO protein remains unchanged (Tang et al., 2014).

The toprim domain consists of four stranded parallel $\beta$-sheet flanked by a $\alpha$-helix from one side and two $\alpha$-helices from the other side (Fig. 4a). This represents a typical Rossmann type nucleotide-binding fold. The toprim domain is also present in DNA binding proteins such as Topoisomerase (types IA and II), DnaG-type primases and old family nucleases. The toprim domain comprised of a conserved glutamic acid residue at the start of the toprim domain and a DxD motif towards the end of the domain. The glutamic acid may act as a general base in nucleotide polymerization by primase and stand ligation by topoisomerase, whereas in case of strand cleavage by topoisomerase and nuclease, as a general acid (Aravind et al., 1998). The DxD motif is required for the binding of the $\mathrm{Mg}^{2+}$ ion and is important for the function of all toprimcontaining enzymes. The glutamic acid was found to be partially conserved and DxD motif is absent in RecR proteins (Allemand et al., 2005; Aravind et al., 1998; Fu et al., 2009). Thus, the toprim domain of RecR may not act as those seen in topoisomerase and primase enzymes. However, it can perform an important role in DNA binding and recombination mediator protein interactions.

The Walker-B motif and the preceding $\alpha$-helix take part in domain swap at the C-terminal and help in forming a tetramer (Fig. 4b). The C-terminal deleted mutants of DrRecR showed aggregation during purification (Lee et al., 2004), whereas TtRecR showed a dimeric population. However, the N-terminal deletion of TtRecR was a monomer. This showed that it is the N-terminal and not the C-terminal that is required for stable dimer formation (Honda et al., 2008; Tang et al., 2012).

\section{Tetramerization of TtRecR protein}

Both TtRecR and EcRecR are known to form dimers in solution. However, a homo-tetrameric assembly was observed in the crystal structures of TtRecR, DrRecR and TteRecR. The RecR protein interacts with RecF and RecO proteins in tetrameric state (Honda et al., 2008). However, the factors responsible for the tetramerization of RecR are not known. Also, the interactions of RecR with other cognate proteins for binding to the DNA are not clear. Earlier studies showed that the N-terminal of RecR is required for stable dimer formation and not the C-terminal (Honda et $a l ., 2006)$. Thus, the swap region of the $\mathrm{C}$-terminal was targeted for cysteine mutation to form a stable tetramer in solution. Gly169 present at the C-terminus of TtRecR is mutated to cysteine 
(Fig. 4b). The purification of TtRecR ${ }_{\mathrm{G} 169 \mathrm{C}}$ mutant resulted in a dimeric $44.4 \mathrm{kDa}$ band along with a small quantity of monomeric band at $22.2 \mathrm{kDa}$ (Fig. 3). This concludes that the TtRecR $\mathrm{G}_{169 \mathrm{C}}$ mutant exists as a tetramer in solution even at very low concentration. The stable tetrameric TtRecR has been tested for its interaction with ssDNA and other repair mediator proteins.

\section{Stabilized tetrameric mutant of TtRecR shows distinct interaction properties}

The RecR protein interacts with both the RecF and RecO proteins by forming either RecOR or RecFR complexes. The structural studies on RecOR complex from D. radiodurans showed that the complex consists of a heterohexamer in a ratio of 4RecR:2RecO (PDB id - 4JCV) (Timmins et al., 2007). The tetrameric DrRecR is bound with two monomeric RecO proteins at the adjacent sides of its central cavity. The OB-fold domain of the $\mathrm{RecO}$ protein is pointed towards the interior of the DrRecR cavity. The DrRecOR complex shows preferential binding towards the 3' overhang of ssDNA-dsDNA hybrid. The site directed mutagenesis studies on DrRecR and DrRecO showed that the conserved Glu146 residue present in the toprim domain of DrRecR (equivalent residue; Glu144 in TtRecR) and His93 of DrRecO (equivalent residue not present in TtRecO) are essential for the formation of this complex (Timmins et al., 2007). Structural and biochemical studies on the RecOR complex from $T$. tengcongensis also showed that the loop region (106 to 121) present in the toprim domain of RecR is essential for RecO binding (Tang et al., 2012).

RecR can also form a complex with the RecF protein. The TtRecFR complexes are formed in 4:2 ratio (4TtRecR:2ttRecF) (Honda et al., 2008). Both, the N-terminal and the C-terminal of TtRecR are required for the TtRecFR complex formation. The TtRecR interacts with the TtRecF in a tetrameric state. The SASX data suggests that the assembly of this hetero-hexameric complex is such that the two RecF can interact with tetrameric TtRecR molecules, either on the opposite sides of the central RecR cavity or from the same side. However, in both the cases, the central cavity of the tetrameric TtRecR was covered by the TtRecF proteins, leaving no space for DNA to pass (Honda et al., 2008). The mutation of Glu144 residue in TtRecR showed reduced binding to TtRecO protein (as also observed in the equivalent mutation in DrRecR) and completely abolished its interaction with TtRecF. Thus, both (TtRecF and TtRecO) proteins share an overlapping binding site for their binding to the TtRecR protein and both the proteins interacts with the tetrameric RecR (Honda et al., 2006). 
To elucidate the effect of the TtRecR tetramerization (TtRecR $\mathrm{G}_{169 \mathrm{C}}$ ) on the binding of the wild type TtRecF and TtRecO proteins, Native-PAGE and EMSA experiments were performed. The proteins $\mathrm{TtRecF}$ and $\mathrm{TtRecO}$ have higher $\mathrm{pI}$ values of 8.92 and 10.25, respectively as compared to RecR (pI 5.43). Thus, TtRecF and TtRecO were not able to move in the native-PAGE. An additional band appeared, when TtRecR and TtRecR $\mathrm{G}_{169 \mathrm{C}}$ were incubated with the TtRecF protein, suggesting a stable TtRecFR/TtRecFR ${ }_{\mathrm{G} 169 \mathrm{C}}$ complex formation in solution (Figs. 6a and 6b). Nevertheless, the TtRecOR complex formation should not be neglected. The TtRecR/TtRecR ${ }_{\text {G169C }}$ interactions may not be able to subside the positive charge on the TtRecO protein and hence the complex remains in the well during the electrophoresis. This suggest that the interactions of TtRecF and TttRecO proteins with the wild type and mutant TtRecR would be similar. Further, these interactions were also studied in the presence of fluorescein (FAM) labelled ssDNA. The

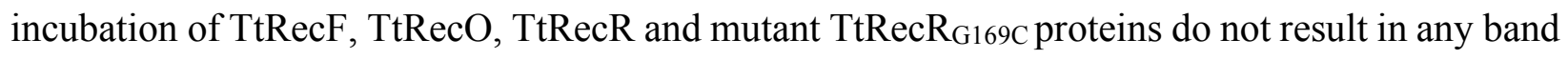
shift. A shift in the ssDNA band was observed when the TtRecO or TtRecF proteins were incubated with the wild type TtRecR, whereas, these shifts were missing in the presence of the mutant, TtRecR ${ }_{\mathrm{G} 169 \mathrm{C}}$ (Figs. 7a and 7b).

\section{TtRecO displays higher binding to ssDNA in the presence of the wild type TtRecR}

The individual binding affinities of the wild type and mutant proteins with ssDNA have been monitored using Bio-Layer Interferometry (BLI). The wild type TtRecR exhibited negligible binding with the ssDNA, whereas the mutant TtRecR $\mathrm{G}_{\mathrm{G} 169 \mathrm{C}}$ showed binding and the binding data can be fitted using mass transport binding model (Figs. 8a and 8b). However, this has not helped in determining the $\mathrm{K}_{\mathrm{D}}$ value accurately. TtRecO showed a strong binding with the ssDNA (Fig. $8 \mathrm{c})$. The binding curve can be fitted in the heterogeneous ligand model, which suggests that the TtRecO binds to ssDNA with two different off-rate constants; hence RecO might have two different binding modes. A tenfold difference has been recorded between the two binding affinities. The N-terminal OB fold domain of the RecO proteins are mainly responsible for DNA binding, however, the C-terminal domain on the protein was also found to have relatively lesser affinity towards the DNA, as observed in DrRecO (Leiros et al., 2005). This would be the reason for the biphasic behavior of TtRecO binding.

The TtRecO interacts with the TtRecR proteins to form TtRecOR complex in a ratio of 2RecO:4RecR (Timmins et al., 2007). Its interaction with the ssDNA was monitored. A huge jump 
in the nm was observed in the presence of TtRecR and TtRecO (Fig. 8d). This normally means a better binding affinity, however, considering the measurement principle of the instrument, the shift in the interference pattern is the result of changing thickness at the tip of the biosensors. Here, the increase in the thickness would be due to the binding of TtRecOR complex instead of only TtRecO. A higher shift was observed, when the concentration of the wild type TtRecR or the mutant

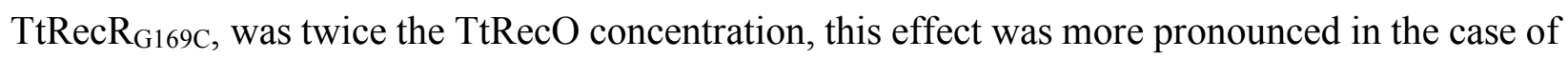
the wild type TtRecR (Fig. 8d). Indicating, that the conformational flexibility between the dimeric TtRecR protein would assist the binding of TtRecO protein. Moreover, a decrease in the shift is observed, when equal concentrations of the TtRecO and TtRecR/RecR $\mathrm{G}_{169 \mathrm{C}}$ proteins were used and this shift was equivalent to the TtRecO shift (Figs. 8c and 8d). This shows a molarity dependent interaction stabilization between the TtRecR and TtRecO proteins.

\section{TtRecF mutants shows affinity for ssDNA}

The native-PAGE has confirmed the formation of TtRecFR and TtRecFR ${ }_{\mathrm{G} 169 \mathrm{C}}$ complexes in the solution. EMSA results showed that TtRecF has no affinity for ssDNA, however a band shift appeared, when incubated with the wild type TtRecR protein. The BLI experiments also complimented these results. Conversely, a slight increase in the $\mathrm{nm}$ shift was observed in the presence of the wild type TtRecR and mutant TtRecR ${ }_{\mathrm{G} 169 \mathrm{C}}$ (Figs. 9a and 9b). These shifts are perhaps due to mass transfer limited kinetics, where the rate of binding to the target site is faster than its diffusion. This shows that the TtRecFR complex has negligible affinity towards the ssDNA as compared to the TtRecOR complex. The RecFR complex has been shown to bind at the junction of gDNA and needs a free 5'end for proper binding (Morimatsu \& Kowalczykowski, 2003). It suggests that the break site could be recognized by the RecFR complex.

Surprisingly, in contrast to the wild type TtRecF, the non-canonical dimers formed by the cysteine mutations ( $\mathrm{TtRecF}_{\mathrm{D} 57 \mathrm{C}}$ and $\left.\mathrm{TtRecF}_{\mathrm{S} 261 \mathrm{C}}\right)$, showed binding towards the ssDNA even in the absence of TtRecR (Figs. 10a and 10b). The binding data can be fitted using heterogeneous ligand method; however the two $\mathrm{K}_{\mathrm{D}}$ values were very similar. Both the mutants $\left(\mathrm{TtRecF}_{\mathrm{D} 57 \mathrm{C}}\right.$ and $\left.\mathrm{TtRecF}_{\mathrm{S} 261 \mathrm{C}}\right)$ showed binding affinities of 146.3 and $19.7 \mu \mathrm{M}$, respectively. A shift in the nm was observed after the addition of the wild type TtRecR. The shift was more pronounced when the concentration of TtRecR proteins was twice the concentration of $\mathrm{TtRecF}_{\mathrm{D} 57 \mathrm{C}} / \mathrm{TtRecF}_{\mathrm{S} 261 \mathrm{C}}$ mutant proteins (Fig. $10 \mathrm{c})$. It complimented the binding ratios of $2 \mathrm{RecF}: 4 \mathrm{Rec} R$ in the complexes as suggested in an 
earlier study (Honda et al., 2006). In contrast to the wild type TtRecF, these non-canonical dimeric $\mathrm{TtRecF}_{\mathrm{D} 57 \mathrm{C}} / \mathrm{TtRecF}_{\mathrm{S} 261 \mathrm{C}}$ mutants can bind to only one face of the TtRecR. This suggests that the binding topology of RecF to RecR can regulate the complex affinity towards the ssDNA and dsDNA. The dsDNA binding property of the RecFR complex is important for the DNA break recognition, whereas the physiological significance of the ssDNA binding needs to be explored.

\section{TtRecO shows higher affinity for TtSSB bound ssDNA in the presence of tetrameric TtRecR}

As soon as the break is formed in the DNA, the helicase and endonuclease activities of RecQ and RecJ, respectively, creates a 3' overhang. This 3' overhang is immediately coated by the SSB proteins. The binding affinity $\left(\mathrm{K}_{\mathrm{D}}\right)$ of the TtSSB proteins for the ssDNA is $7.2 \mathrm{nM}$ (Fig. 11), which is approximately thousand times higher than that of the TtRecO protein $(2.4 \mu \mathrm{M})$. Upon performing the TtSSB association step, a minor dissociation was observed. Strong binding property of TtSSB to ssDNA was further used to monitor the binding of other RecFOR pathway proteins on the TtSSB coated ssDNA and another step of association and dissociation was added to the experiments.

The wild type TtRecR, TtRecR $\mathrm{G}_{169 \mathrm{C}}$ and TtRecFR complex showed a negligible association to the TtSSB bound ssDNA (Fig. 12a and 12b). However, when the TtSSB coated ssDNA was incubated with the TtRecO protein, an increase in the nm shift was observed (Fig. 12c). This indicates the binding of the TtRecO protein on the TtSSB bound ssDNA. Structural studies on the EcSSB showed that the SSB C-terminal (SSB-Ct) has affinity for the EcRecO protein, which in turn help in its recruitment on the SSB coated ssDNA (Inoue et al., 2011). The C-terminal sequence of TtSSB ("EEELPF") is similar to the EcSSB ("DDDIPF”). Both the TtSSB and TtRecO proteins carry OB folds, required for DNA binding, however, the dimeric TtSSB carry four OB folds as compared to only one OB fold in the monomeric TtRecO. Thus, the binding of two TtRecO proteins at both the C-termini of the TtSSB may result in the decrease in the affinity of the TtSSB protein with the ssDNA. An increased nm shift was observed, when the SSB bound ssDNA was incubated with the TtRecO and TtRecR/TtRecR ${ }_{\text {G169C }}$ proteins (Fig. 12d). The nm shift was more pronounced when the concentration of $T t R e c R / T t R e c R_{\text {G169C }}$ proteins was twice than that of the TtRecO concentration. In contrast to wild type TtRecR binding to TtSSB coated ssDNA, the mutant tetrameric TtRecR ${ }_{\mathrm{G} 169 \mathrm{C}}$ showed a higher nm shift in the presence of TtRecO. This suggests that the tetrameric state of TtRecR in complex with TtRecO has preferential binding towards the 
TtSSB coated ssDNA. However, binding of these complexes did not lead to the release of TtSSB from ssDNA. Nevertheless, the TtRecOR complex may reduce the binding affinity of the TtSSB with ssDNA and help in the recruitment of TtRecA protein. The TtRecA proteins forms a nucleoprotein filament on the ssDNA by displacing the SSB and RecOR complex and prepares the DNA for strand exchange.

\section{Conclusion}

Condition dependent stabilization of homomeric or heteromeric complexes plays a major role in regulating many major pathways in the cells. However, these complexes are very transient, which makes it difficult to study their function. Hence, one way to stabilize is through the di-sulphide bonds. RecO and RecF share a common interaction site on the RecR protein and form a heterohexameric complexes in a ratio $2 \mathrm{RecO} / \mathrm{RecF}: 4 \mathrm{RecR}$. TtRecR exists as a dimer in solution; conversely, the crystal structure supports a tetrameric arrangement of TtRecR. The tetrameric state of TtRecR was stabilized by mutating the glycine residue at the domain swap region of the Cterminal. This resulted in both the dimeric as well as tetrameric populations in the solution. This points out that the TtRecR exist in equilibrium between dimer and tetramer in solution, however as seen in the crystal structure, with the increase in concentration, the equilibrium shifts towards the tetrameric state. In comparison to the wild type TtRecR, the mutant tetrameric TtRecR showed affinity towards the ssDNA, suggesting a different mode of interaction.

TtRecF exists as a monomer in solution, it dimerizes in the presence of ATP and shows DNA dependent ATP hydrolysis. Cysteine mutations were introduced in the two interface residues of the modeled dimeric TtRecF to stabilize its dimeric state. Surprisingly, the TtRecF cysteine mutants formed a non-canonical dimer during their purification. Interestingly, in contrast to the wild type TtRecF, the non-canonical dimeric TtRecF mutants showed affinity towards ssDNA and a concentration dependent affinity change was observed in the presence of wild type TtRecR. Interestingly, wild type RecF showed no affinity towards ssDNA. Thus, suggesting a distinct interaction mode of the TtRecF protein with the ssDNA and TtRecR protein.

TtSSB and TtRecO proteins showed affinity towards the ssDNA, nonetheless, TtSSB has hundredfold more binding affinity than that of TtRecO. The TtSSB bound ssDNA exhibited negligible 
dissociation and hence is used as a template to study the binding of other cognate proteins. Individually, the wild type TtRecR and the tetrameric TtRecR or in complex with the TtRecF has no affinity for the SSB coated ssDNA. On the other hand, TtRecO binds with almost equal affinity with the TtSSB coated ssDNA as observed with the ssDNA. A higher nm shift is recorded in the presence of TtRecR/TtRecR ${ }_{\mathrm{G} 169 \mathrm{C}}$ by forming $\mathrm{TtRecOR} / \mathrm{TtRecOR}_{\mathrm{G} 169 \mathrm{C}}$ complexes. This shift is more prominent in the case of the $\mathrm{TtRecOR}_{\mathrm{G} 169 \mathrm{C}}$ suggesting the importance for the tetrameric state of TtRecR in stabilizing hetero-hexameric complex and also its binding to SSB coated ssDNA.

Further, a model for SSB dissociation from ssDNA can be proposed based on our studies on protein-protein and protein-DNA interactions. The TtSSB exists as a dimer in solution and binds to the ssDNA using four OB fold domains. The TtRecO protein interacts with the C-terminal of TtSSB and has a single OB fold. The dimeric TtSSB can bind at least two TtRecO proteins and the presence of two OB folds may reduce the affinity of TtSSB for the ssDNA. This phenomenon is further reinforced by the presence of TtRecR forming the TtRecOR complex. However, our experiments suggest that the reduced affinity is not sufficient for the release of TtSSB protein from ssDNA. Nevertheless, it would be sufficient for the recruitment of the RecA protein, which may replace the bound SSB and RecOR complex by forming a nucleoprotein filament on the ssDNA.

\section{ACKNOWLEDGEMENTS}

The authors thank Department of Science and Technology (DST) for funding. The authors thank the Department of Biotechnology (DBT), Govt. of India and the facilities offered by the center of excellence in structural biology and bio-computing, Supercomputer Education and Research Center and Department of Computational and Data Sciences, Indian Institute of Science, Bangalore. The authors thank ESRF BM-14 beam line for data collection.

Disclosure Statement: The authors declare that they have no competing financial interests. 


\section{$\underline{\text { References }}$}

Allemand, F., Mathy, N., Brechemier-Baey, D. \& Condon, C. (2005).Nucleic Acids Res 33(13): 4368-4376.

Aravind, L., Leipe, D. D. \& Koonin, E. V. (1998).Nucleic Acids Res 26(18): 4205-4213.

Baker, N. A., Sept, D., Joseph, S., Holst, M. J. \& McCammon, J. A. (2001).Proc Natl Acad Sci U $S$ A 98(18): 10037-10041.

Bidnenko, V., Seigneur, M., Penel-Colin, M., Bouton, M. F., Dusko Ehrlich, S. \& Michel, B. (1999).Mol Microbiol 33(4): 846-857.

Doherty, A. J., Serpell, L. C. \& Ponting, C. P. (1996).Nucleic Acids Res 24(13): 2488-2497.

Fu, G., Wu, J., Liu, W., Zhu, D., Hu, Y., Deng, J., Zhang, X. E., Bi, L. \& Wang, D. C. (2009).Nucleic Acids Res 37(17): 5908-5916.

Gupta, R., Shuman, S. \& Glickman, M. S. (2015).J Bacteriol 197(19): 3121-3132.

Handa, N., Morimatsu, K., Lovett, S. T. \& Kowalczykowski, S. C. (2009).Genes Dev 23(10): 1234-1245.

Hanson, P. I. \& Whiteheart, S. W. (2005).Nat Rev Mol Cell Biol 6(7): 519-529.

Harmon, F. G. \& Kowalczykowski, S. C. (1998).Genes Dev 12(8): 1134-1144.

Holm, L. \& Laakso, L. M. (2016).Nucleic Acids Res 44(W1): W351-355.

Honda, M., Fujisawa, T., Shibata, T. \& Mikawa, T. (2008).Nucleic Acids Res 36(15): 5013-5020.

Honda, M., Inoue, J., Yoshimasu, M., Ito, Y., Shibata, T. \& Mikawa, T. (2006).J Biol Chem 281(27): 18549-18559.

Inoue, J., Nagae, T., Mishima, M., Ito, Y., Shibata, T. \& Mikawa, T. (2011).J Biol Chem 286(8): 6720-6732.

Koroleva, O., Makharashvili, N., Courcelle, C. T., Courcelle, J. \& Korolev, S. (2007).EMBO J 26(3): 867-877.

Kusakabe, T., Hine, A. V., Hyberts, S. G. \& Richardson, C. C. (1999).Proc Natl Acad Sci U S A 96(8): 4295-4300.

Lee, B. I., Kim, K. H., Park, S. J., Eom, S. H., Song, H. K. \& Suh, S. W. (2004).EMBO J 23(10): 2029-2038.

Lee, H., Park, H., Ko, J. \& Seok, C. (2013).Bioinformatics 29(8): 1078-1080. 
Lehmann, A. R. (2005).DNA Repair (Amst) 4(3): 309-314.

Leiros, I., Timmins, J., Hall, D. R. \& McSweeney, S. (2005).EMBO J 24(5): 906-918.

Lovett, S. T. \& Kolodner, R. D. (1989).Proc Natl Acad Sci U S A 86(8): 2627-2631.

Lyskov, S. \& Gray, J. J. (2008).Nucleic Acids Res 36(Web Server issue): W233-238.

McEntee, K., Weinstock, G. M. \& Lehman, I. R. (1979).Proc Natl Acad Sci U S A 76(6): 26152619.

Michel-Marks, E., Courcelle, C. T., Korolev, S. \& Courcelle, J. (2010).J Mol Biol 401(4): 579589.

Moncalian, G., Lengsfeld, B., Bhaskara, V., Hopfner, K. P., Karcher, A., Alden, E., Tainer, J. A. \& Paull, T. T. (2004).J Mol Biol 335(4): 937-951.

Moretti, R., Lyskov, S., Das, R., Meiler, J. \& Gray, J. J. (2018).Protein Sci 27(1): 259-268.

Morimatsu, K. \& Kowalczykowski, S. C. (2003).Mol Cell 11(5): 1337-1347.

Rojowska, A., Lammens, K., Seifert, F. U., Direnberger, C., Feldmann, H. \& Hopfner, K. P. (2014).EMBO J 33(23): 2847-2859.

Shibata, T., Cunningham, R. P., DasGupta, C. \& Radding, C. M. (1979).Proc Natl Acad Sci U S A 76(10): 5100-5104.

Sultana, A. \& Lee, J. E. (2015).Curr Protoc Protein Sci 79: 1925 11-26.

Tang, Q., Gao, P., Liu, Y. P., Gao, A., An, X. M., Liu, S., Yan, X. X. \& Liang, D. C. (2012).Nucleic Acids Res 40(21): 11115-11125.

Tang, Q., Liu, Y. P., Shan, H. H., Tian, L. F., Zhang, J. Z. \& Yan, X. X. (2018).Sci Rep 8(1): 2127.

Tang, Q., Liu, Y. P., Yan, X. X. \& Liang, D. C. (2014).DNA Repair (Amst) 24: 10-14.

Thayer, M. M., Ahern, H., Xing, D., Cunningham, R. P. \& Tainer, J. A. (1995).EMBO J 14(16): 4108-4120.

Timmins, J., Leiros, I. \& McSweeney, S. (2007).EMBO J 26(13): 3260-3271.

Webb, B. L., Cox, M. M. \& Inman, R. B. (1999).J Biol Chem 274(22): 15367-15374.

Xu, H. \& Freitas, M. A. (2009).Proteomics 9(6): 1548-1555. 


\section{Figure legends}

Figure 1. Crystal structure of RecF in monomeric and its modelled dimeric state. (A) Cartoon representation of RecF with individual motifs labelled in distinct colors. The ATPase and DNA binding domains are represented at the side of the structure. (B) The dimeric interface of the RecF was modelled using Rosetta server. The $\mathrm{C} \alpha$ atom of the residues Ser261 and Asp57 are present at a distance of $6 \AA$. These residues were selected for mutation to cysteine residues ( $\mathrm{TtRecF} \mathrm{D}_{\mathrm{D} 5 \mathrm{C}}$ and

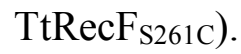

Figure 2. Structure based multiple sequence alignment of RecF proteins. The RecF sequences are taken from various organisms (marked at the beginning). Highly conserved regions are marked in red and their names are mentioned at the base of the sequence.

Figure 3. SDS-PAGE analysis of $\mathrm{TtRecF}_{\mathrm{D} 57 \mathrm{C}}, \mathrm{TtRecF}_{\mathrm{S} 261 \mathrm{C}}$ and $\mathrm{TtRec}_{\mathrm{G} 169 \mathrm{C}}$ proteins upon treatment with TCEP (tris(2-carboxyethyl)phosphine). TtRecF ${ }_{\mathrm{D} 57 \mathrm{C}}, \mathrm{TtRecF}_{\mathrm{S} 261 \mathrm{C}}$

Figure 4. Monomeric and tetrameric assemblies of the wild-type TtRecR protein. (A) Crystal structure of the wild-type ttRecR. The motifs in the structures are marked in different colors and their names are represented in similar colors. The blue colored sphere represents a Zinc ion. (B) A tetrameric assembly of the wild-type ttRecR is generated by combining the symmetry mates. The $\mathrm{N}$ - and C-terminals of the protein are involved in a domain swap and form a stable tetrameric assembly. The tetrameric assembly creates a central hole of 30-35 A. The Gly169 present at the

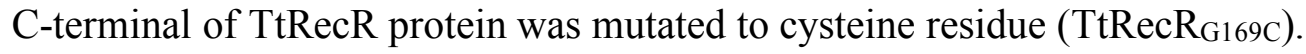

Figure 5. Structure based sequence alignment of RecR proteins. The RecR sequences are taken from various organisms (marked at the beginning of sequences). Highly conserved regions are marked in red and motifs are underlined with their names marked the base.

Figure 6. Analysis of protein-protein interactions $\mathrm{RecF}$ and $\mathrm{RecO}$ with the wild type RecR and mutant $t \mathrm{tRec} \mathrm{R}_{\mathrm{G} 169 \mathrm{C}}$ using native-PAGE. The higher $\mathrm{pI}$ values of $\mathrm{RecO}(\mathrm{pI}-10.3)$ and $\mathrm{RecF}(\mathrm{pI}-$ 8.3) restrict its mobility in the native-PAGE (lanes 1-2), whereas the mobility of RecR/RecR $\mathrm{G}_{169 \mathrm{C}}$ is facilitated by lower $\mathrm{pI}$ value ( $\mathrm{pI}-6.3$ ) (lane 3). $10 \mu \mathrm{M}$ of $\mathrm{RecO}, \mathrm{RecF}$ and $\mathrm{RecR} / \mathrm{RecR} \mathrm{R}_{\mathrm{G} 169 \mathrm{C} \text { was }}$ loaded in the lanes 1-3. (A) $10 \mu \mathrm{M}$ of RecR was incubated with 10, 20 and $40 \mu \mathrm{M}$ concentrations of RecO (lanes 4-6) and with 10, 20 and $40 \mu \mathrm{M}$ concentrations of RecF (lanes 7-9). (B) $10 \mu \mathrm{M}$ of mutant RecR $\mathrm{G}_{169 \mathrm{C}}$ was incubated with 10,20 and $40 \mu \mathrm{M}$ concentrations of RecO (lanes 4-6) and with 10, 20 and $40 \mu \mathrm{M}$ concentrations of RecF (lanes 7-9).

Figure 7. Analysis of protein-protein and protein-ssDNA interactions of RecF and RecO with the wild type RecR and mutant ttRecR $\mathrm{G}_{169 \mathrm{C}}$ using EMSA. Fluorescein (FAM) labelled $\mathrm{dT}_{35}$ was used as ssDNA for the interaction studies. (A) ssDNA was incubated with the $10 \mu \mathrm{M}$ of RecR, RecO and RecF (lanes 2-4). ssDNA was incubated with $10 \mu \mathrm{M}$ of RecR and 5, 10, 20 and $40 \mu \mathrm{M}$ of RecO (lanes 5-8). ssDNA was incubated with $10 \mu \mathrm{M}$ of RecR and 5, 10, 20 and $40 \mu \mathrm{M}$ of RecF (lanes 9-12). (B) ssDNA was incubated with the $10 \mu \mathrm{M}$ of RecF, RecO and RecR $\mathrm{R}_{169 \mathrm{C}}$ (lanes 2-4). ssDNA was incubated with $10 \mu \mathrm{M}$ of $\operatorname{RecR}_{\mathrm{G} 169 \mathrm{C}}$ and 5, 10 and $20 \mu \mathrm{M}$ of RecO (lanes 5-7). ssDNA was incubated with $10 \mu \mathrm{M}$ of $\operatorname{RecR}_{\mathrm{G} 169 \mathrm{C}}$ and 5, 10 and $20 \mu \mathrm{M}$ of RecF (lanes 8-10). 
Figure 8. Interactions of TtRecR, TtRecR ${ }_{\text {G169C }}$, TtRecO and RecOR/RecOR ${ }_{\text {G169C }}$ complexes with ssDNA. (A) Interactions of ssDNA with different concentrations of the wild type TtRecR. (B) Interactions of ssDNA with different concentrations (colored lines) of the stabilized tetrameric

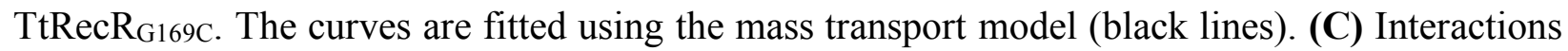
of ssDNA with different concentrations (colored lines) of TtRecO. The curves are fitted using heterogeneous ligand model (Black lines) and the two binding affinities $\left(\mathrm{K}_{\mathrm{D}}\right)$ are marked on it. (D) Interactions of the ssDNA with the TtRecO in the presence of the wild type TtRecR and mutant $\mathrm{TtRecR}_{\mathrm{G169C}}$. The dark green curve denotes incubation of ssDNA with $500 \mathrm{nM}$ of TtRecR and 250 $\mathrm{nM}$ TtRecO. The light green curve denotes incubation of ssDNA with $250 \mathrm{nM}$ of TtRecR and 250 $\mathrm{nM}$ TtRecO. The dark red curve denotes incubation of ssDNA with $500 \mathrm{nM}$ of TtRecR $\mathrm{G}_{169 \mathrm{C}}$ and $250 \mathrm{nM}$ TtRecO. The light red curve denotes incubation of ssDNA with $250 \mathrm{nM}$ of TtRecR $\mathrm{G}_{169 \mathrm{C}}$ and $250 \mathrm{nM}$ TtRecO.

Figure 9. Interactions of TtRecF with ssDNA in the presence and absence of RecR/RecR $\mathbf{G 1 6 9 C}_{\text {. }}$ (A) Interactions of ssDNA with different concentrations of the wild type TtRecF. (B) Interactions of the ssDNA with the TtRecF in the presence of the wild type TtRecR and mutant TtRecR $\mathrm{G}_{6169 \mathrm{C}}$. The dark green curve denotes incubation of ssDNA with $500 \mathrm{nM}$ of TtRecR and $250 \mathrm{nM}$ TtRecF. The light green curve denotes incubation of ssDNA with $250 \mathrm{nM}$ of TtRecR and $250 \mathrm{nM}$ TtRecF. The dark red curve denotes incubation of ssDNA with $500 \mathrm{nM}$ of TtRecR ${ }_{\mathrm{G} 169 \mathrm{C}}$ and $250 \mathrm{nM}$ TtRecF. The light red curve denotes incubation of ssDNA with $250 \mathrm{nM}$ of TtRecR $\mathrm{G}_{169 \mathrm{C}}$ and 250 nM TtRecF.

Figure 10. Interactions of TtRecF mutants with ssDNA in the presence and absence of the wild type RecR. (A) Interactions of ssDNA with different concentrations (colored lines) of

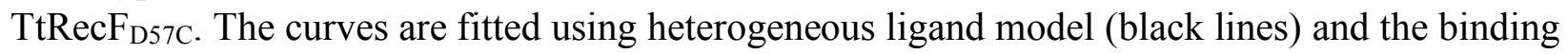
affinity $\left(\mathrm{K}_{\mathrm{D}}\right)$ is marked on it. (B) Interactions of ssDNA with different concentrations (colored lines) of $\mathrm{TtRecF}_{\mathrm{S} 261 \mathrm{C}}$. The curves are fitted using heterogeneous ligand model (black lines) and the binding affinity $\left(\mathrm{K}_{\mathrm{D}}\right)$ is marked on it. (C) Interactions of the ssDNA with the TtRecF $F_{\mathrm{D} 5 \mathrm{C}}$ and TtRecF ${ }_{\text {S261C }}$ in the presence of the wild type TtRecR. The dark green curve denotes incubation of ssDNA with $500 \mathrm{nM}$ of TtRecR and $250 \mathrm{nM}$ TtRecF $\mathrm{D}_{\mathrm{D} 7 \mathrm{C}}$. The light green curve denotes incubation of ssDNA with $250 \mathrm{nM}$ of TtRecR and $250 \mathrm{nM}$ TtRecF ${ }_{\mathrm{D} 57 \mathrm{C}}$. The dark blue curve denotes

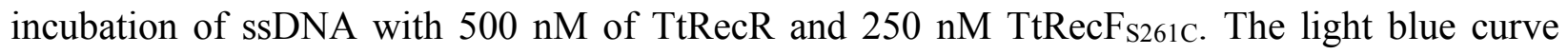
denotes incubation of ssDNA with $250 \mathrm{nM}$ of TtRecR and $250 \mathrm{nM} \mathrm{TtRecF} \mathrm{S}_{\mathrm{S} 61 \mathrm{C}}$.

Figure 11. Interactions of single strand binding protein (SSB) with ssDNA. Interactions of ssDNA with different concentrations of SSB are represented by colored curves. The curves are fitted using 1:1 binding model (black lines) and the binding affinity $\left(\mathrm{K}_{\mathrm{D}}\right)$ is marked on it.

Figure 12. Interactions of SSB coated ssDNA with the RecFOR proteins. (A) Interactions of the wild type RecR (green) and mutant $\operatorname{RecR}_{\mathrm{G} 169 \mathrm{C}}$ (red) with the SSB coated ssDNA. (B) Interactions of SSB coated ssDNA with TtRecF in the presence of the wild type TtRecR and

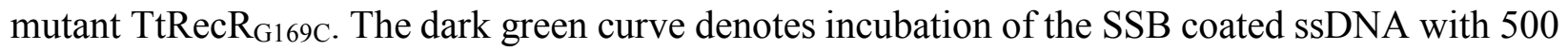
$\mathrm{nM}$ of TtRecR and $250 \mathrm{nM}$ TtRecF. The light green curve denotes incubation of the SSB coated ssDNA with $250 \mathrm{nM}$ of TtRecR and $250 \mathrm{nM}$ TtRecF. The dark red curve denotes incubation of the SSB coated ssDNA with $500 \mathrm{nM}$ of TtRecR $\mathrm{G}_{169 \mathrm{C}}$ and $250 \mathrm{nM}$ TtRecF. The light red curve 
denotes incubation of the SSB coated ssDNA with $250 \mathrm{nM}$ of TtRecR $\mathrm{G}_{\mathrm{G} 169 \mathrm{C}}$ and $250 \mathrm{nM}$ TtRecF. (C) Interactions of the SSB coated ssDNA with different concentrations of TtRecO are represented by colored curves. (D) Interactions of the SSB coated ssDNA with the TtRecO in the presence of

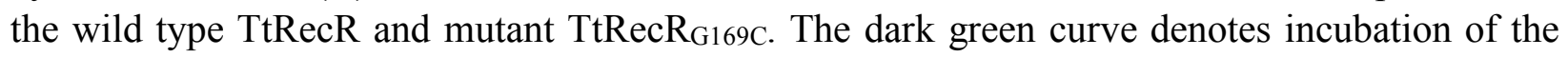
SSB coated ssDNA with $500 \mathrm{nM}$ of TtRecR and $250 \mathrm{nM}$ TtRecO. The light green curve denotes incubation of the SSB coated ssDNA with $250 \mathrm{nM}$ of TtRecR and $250 \mathrm{nM}$ TtRecO. The dark red curve denotes incubation of the SSB coated ssDNA with $500 \mathrm{nM}$ of TtRecR ${ }_{\mathrm{G} 169 \mathrm{C}}$ and $250 \mathrm{nM}$ TtRecO. The light red curve denotes incubation of the SSB coated ssDNA with $250 \mathrm{nM}$ of TtRecR $_{\mathrm{G} 169 \mathrm{C}}$ and $250 \mathrm{nM}$ TtRecO. 


\section{Tables}

Table 1. Data processing and refinement statistics of TtRecF and TtRecR.

\begin{tabular}{|c|c|c|}
\hline Protein name & TtRecF & TtRecR \\
\hline Wavelength $(\AA)$ & 0.95 & 1.54 \\
\hline \multirow[t]{2}{*}{ Resolution limit (Å) } & $51.12-1.58$ & $50.11-2.50$ \\
\hline & $(1.60-1.58)$ & $(2.60-2.50)$ \\
\hline \multirow[t]{3}{*}{ Unit cell dimensions $(\AA)$} & 37.70 & 35.92 \\
\hline & 74.38 & 100.22 \\
\hline & 153.35 & 136.02 \\
\hline $\mathbf{R}_{\text {merge }}(\%)$ & $4.4(35.5)$ & $6.7(89.5)$ \\
\hline \# of Unique reflections & 60237 & 8602 \\
\hline \# of molecules in the & 1 & 1 \\
\hline \multicolumn{3}{|l|}{ asymmetric unit } \\
\hline Completeness $(\%)$ & $99.3(82.0)$ & $96.8(93.6)$ \\
\hline Multiplicity & $4.8(3.8)$ & $10.3(9.1)$ \\
\hline $\operatorname{Mean}((I) / \mathbf{s d}(I))$ & $17.2(3.5)$ & $14.6(1.5)$ \\
\hline \multicolumn{3}{|c|}{ Refinement statistics } \\
\hline $\mathbf{R}_{\text {cryst }} / \mathbf{R}_{\text {free }}(\%)$ & $17.0 / 22.0$ & $23.1 / 28.0$ \\
\hline rmsd bond length $(\AA)$ & 0.019 & 0.007 \\
\hline rmsd bond angle $\left(^{\circ}\right)$ & 1.97 & 1.20 \\
\hline \multicolumn{3}{|c|}{ Average B-factors $\left(\AA^{2}\right)$} \\
\hline Protein & 31.58 & 76.78 \\
\hline Water & 38.17 & 63.65 \\
\hline Ligands & - & 65.48 \\
\hline \multicolumn{3}{|c|}{ Ramachandran statistics (\%) } \\
\hline Favored region & 97.3 & 97.4 \\
\hline Allowed region & 2.4 & 2.1 \\
\hline Outliers & 0.3 & 0.5 \\
\hline PDB ID & $5 Z W U$ & 5ZVQ \\
\hline
\end{tabular}

The data collection statistics values for the highest resolution shell are given in parentheses 
bioRxiv preprint doi: https://doi.org/10.1101/2019.12.17.877662; this version posted December 18, 2019. The copyright holder for this preprint (which was not certified by peer review) is the author/funder. All rights reserved. No reuse allowed without permission.

\section{Figures}

Figure 1.
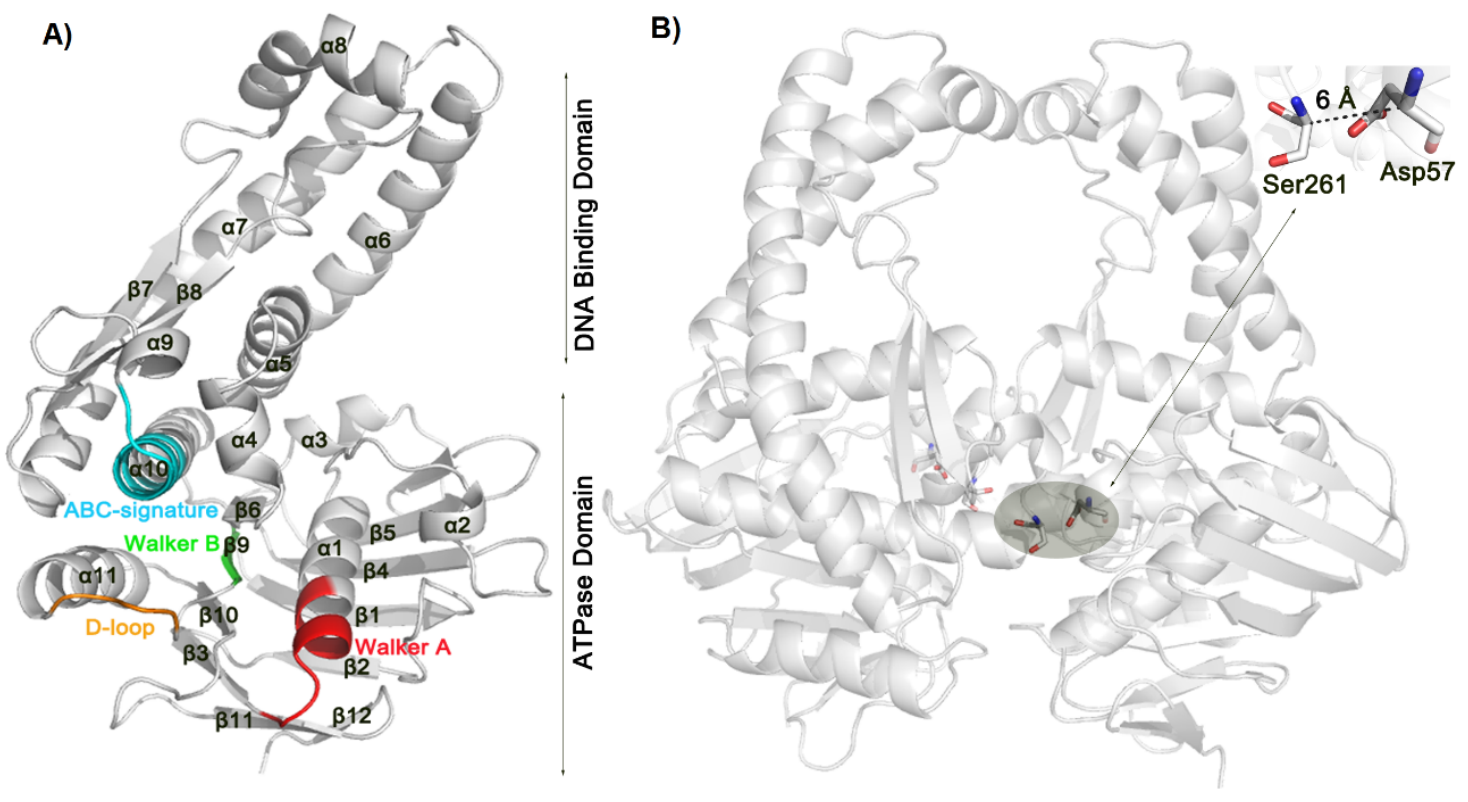


\section{Figure 2.}
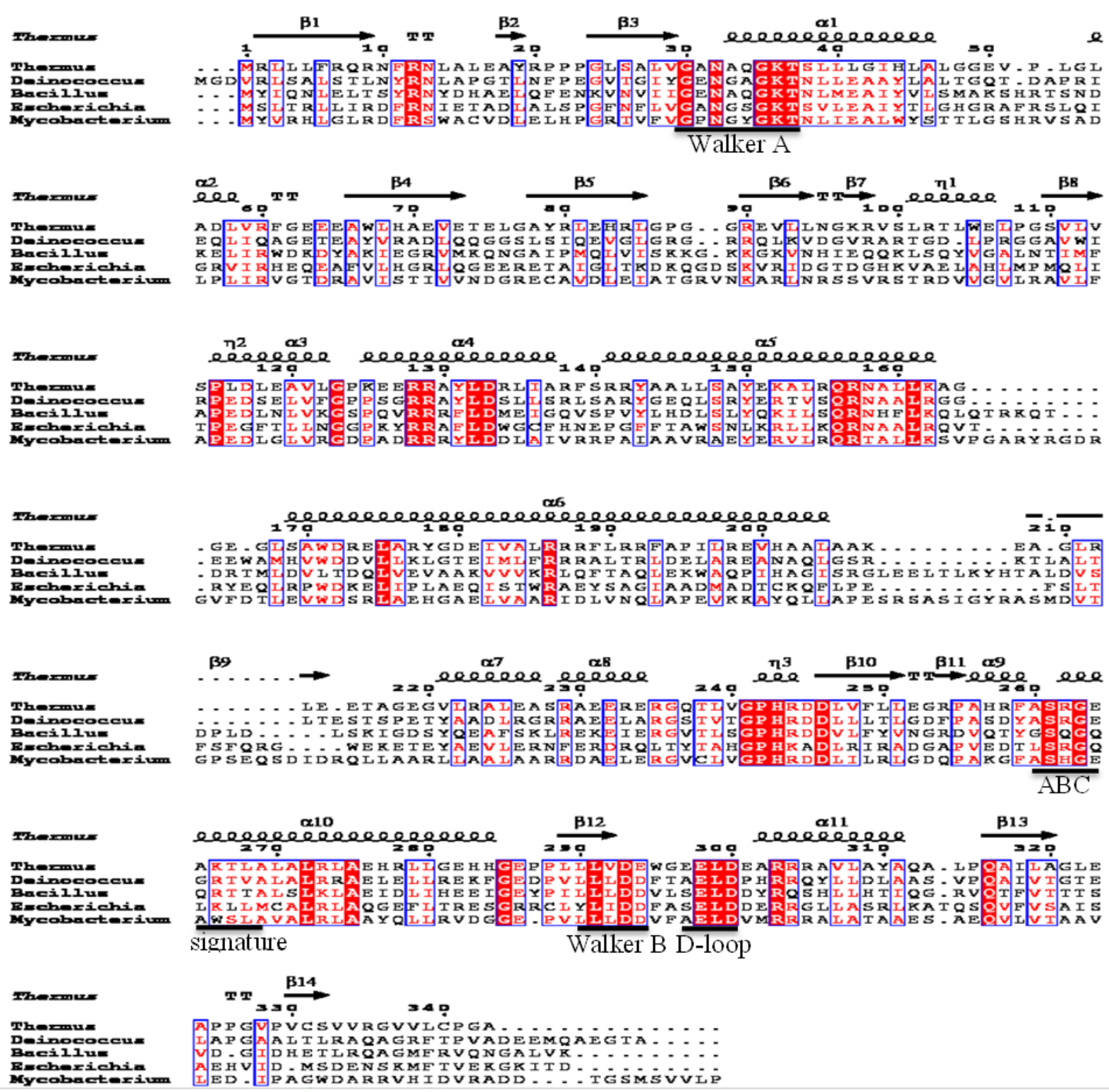
Figure 3.
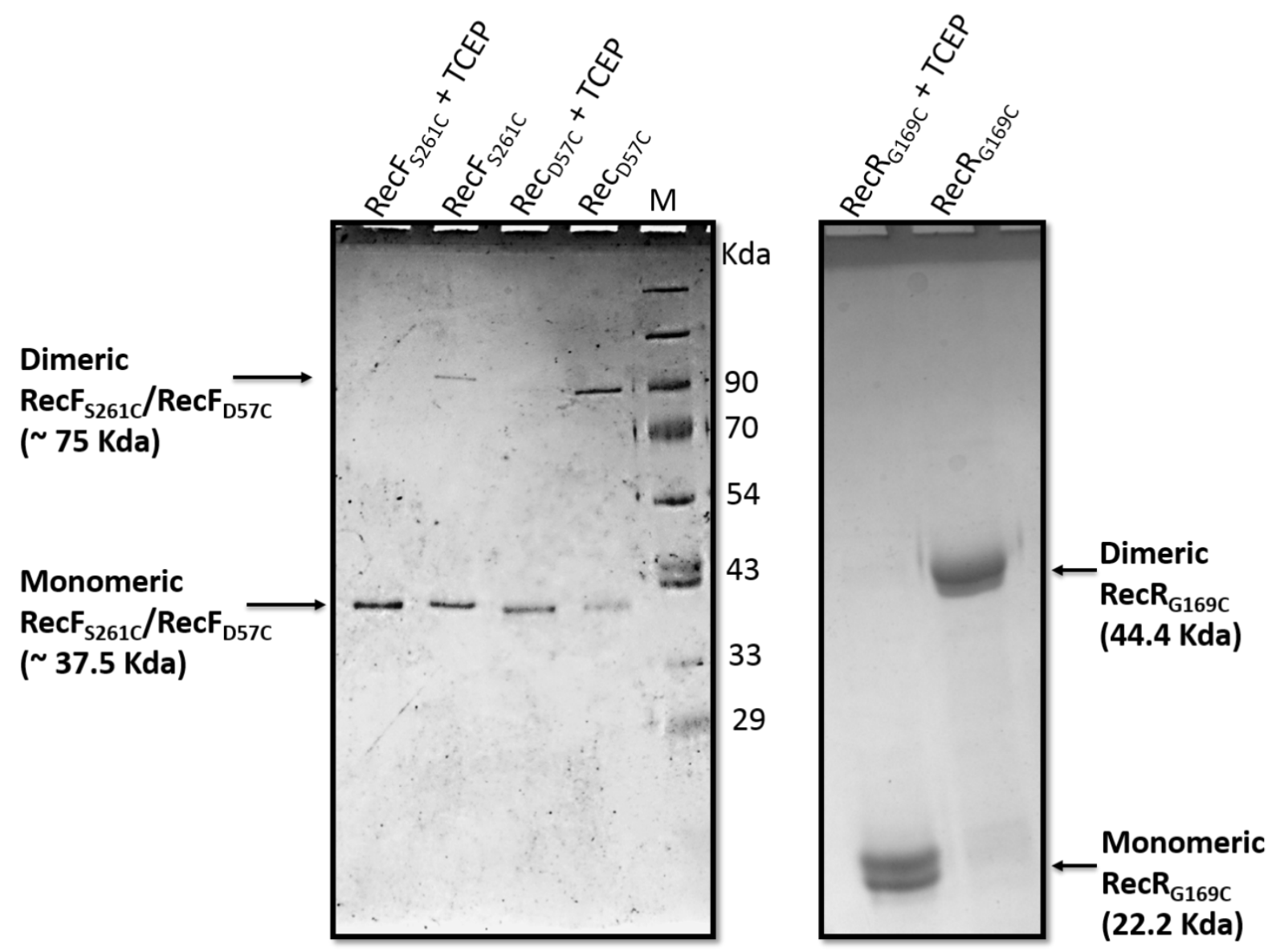
Figure 4.
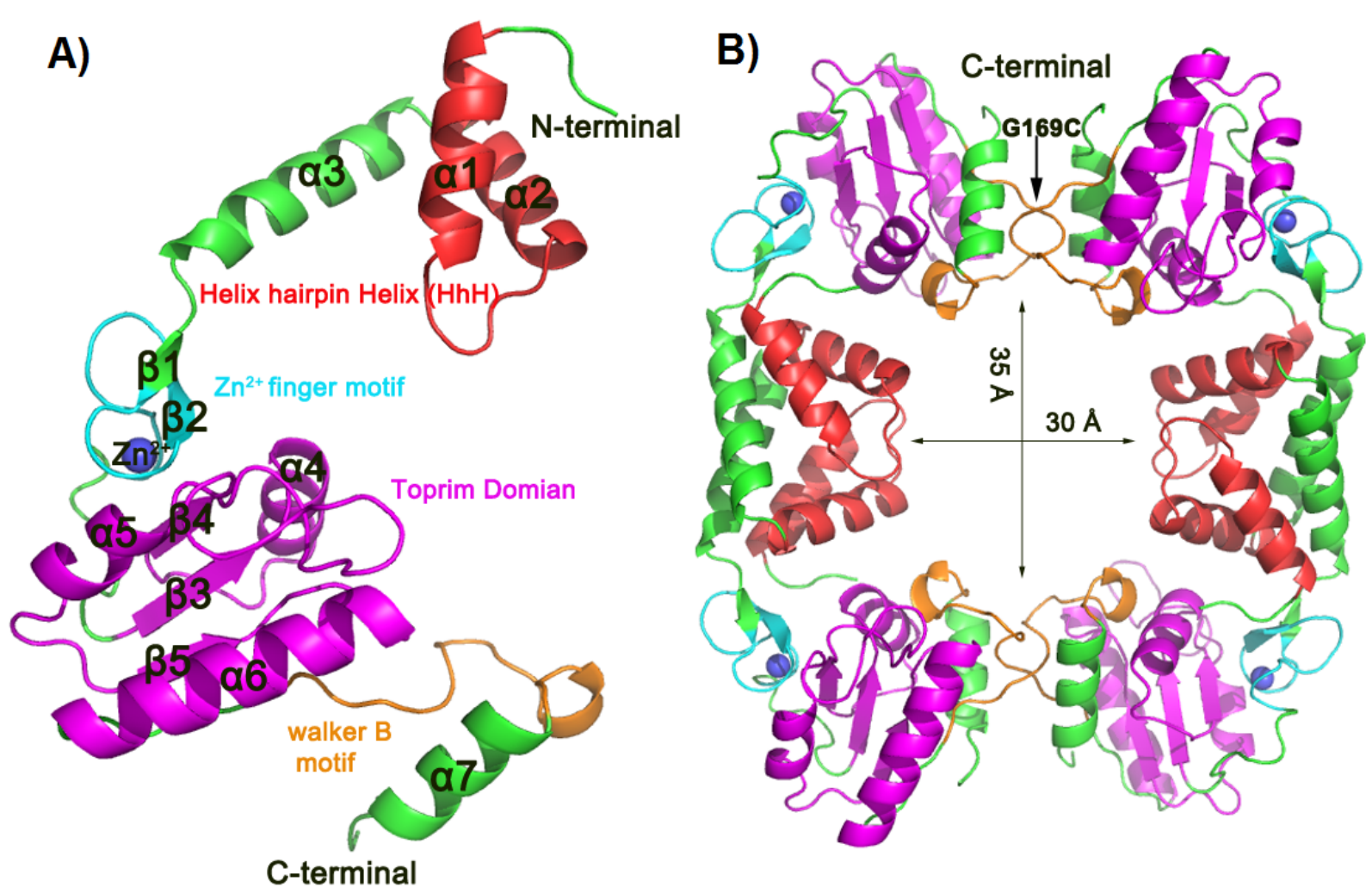


\section{Figure 5.}

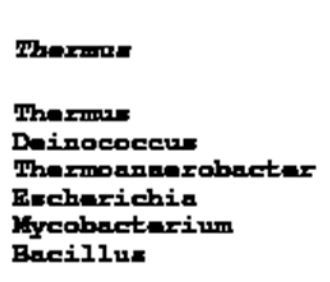

Mherwate

Thermas:

Deinococcur

Thermoanuerobacter

Encherichia

Mycobacterium

Bacillua

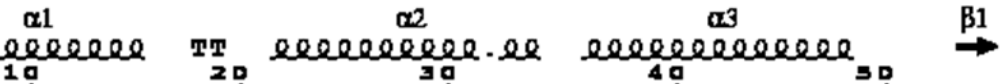

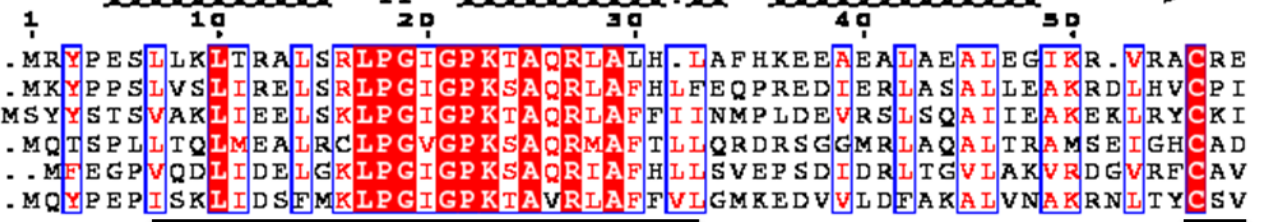

$\mathrm{HhH}$ motif

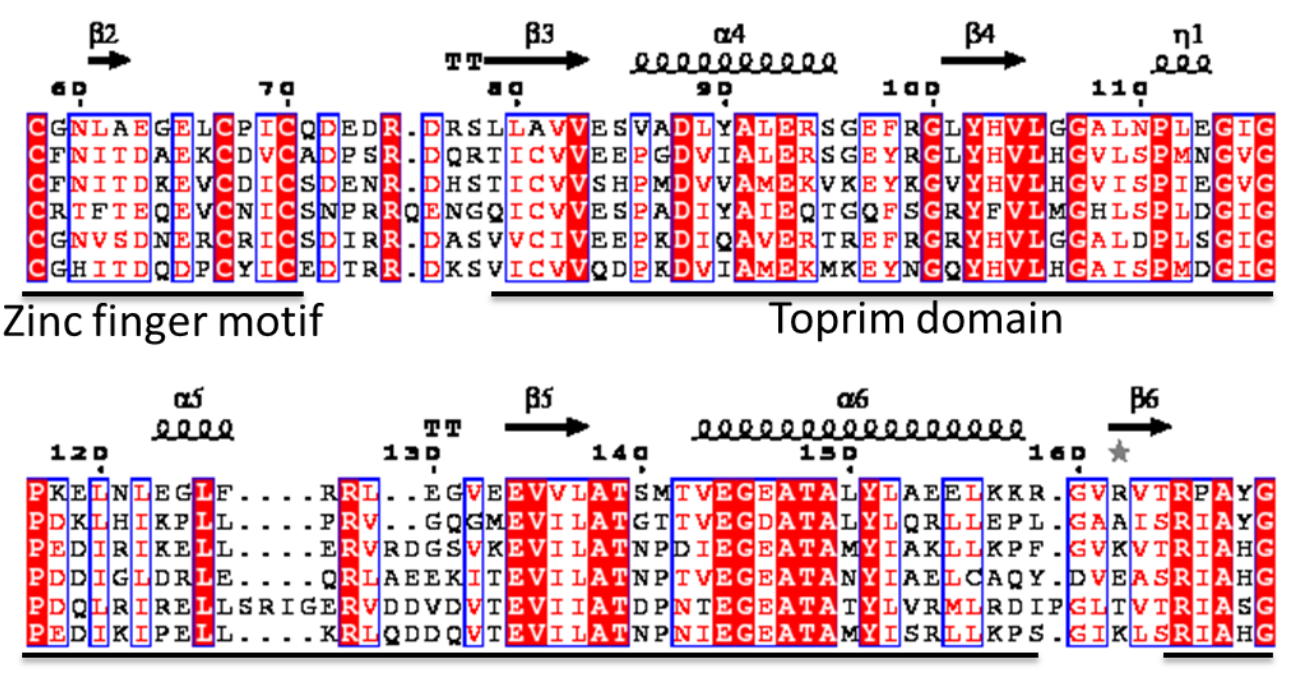

a 7

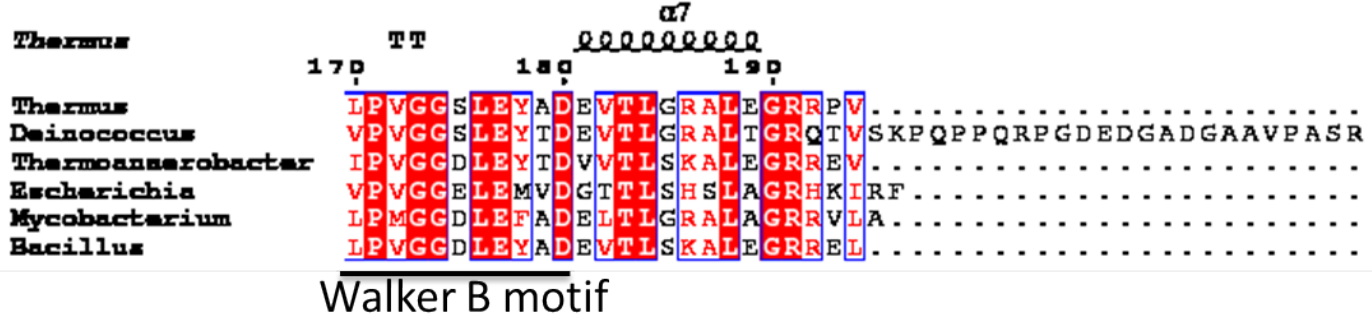




\section{Figure 6.}
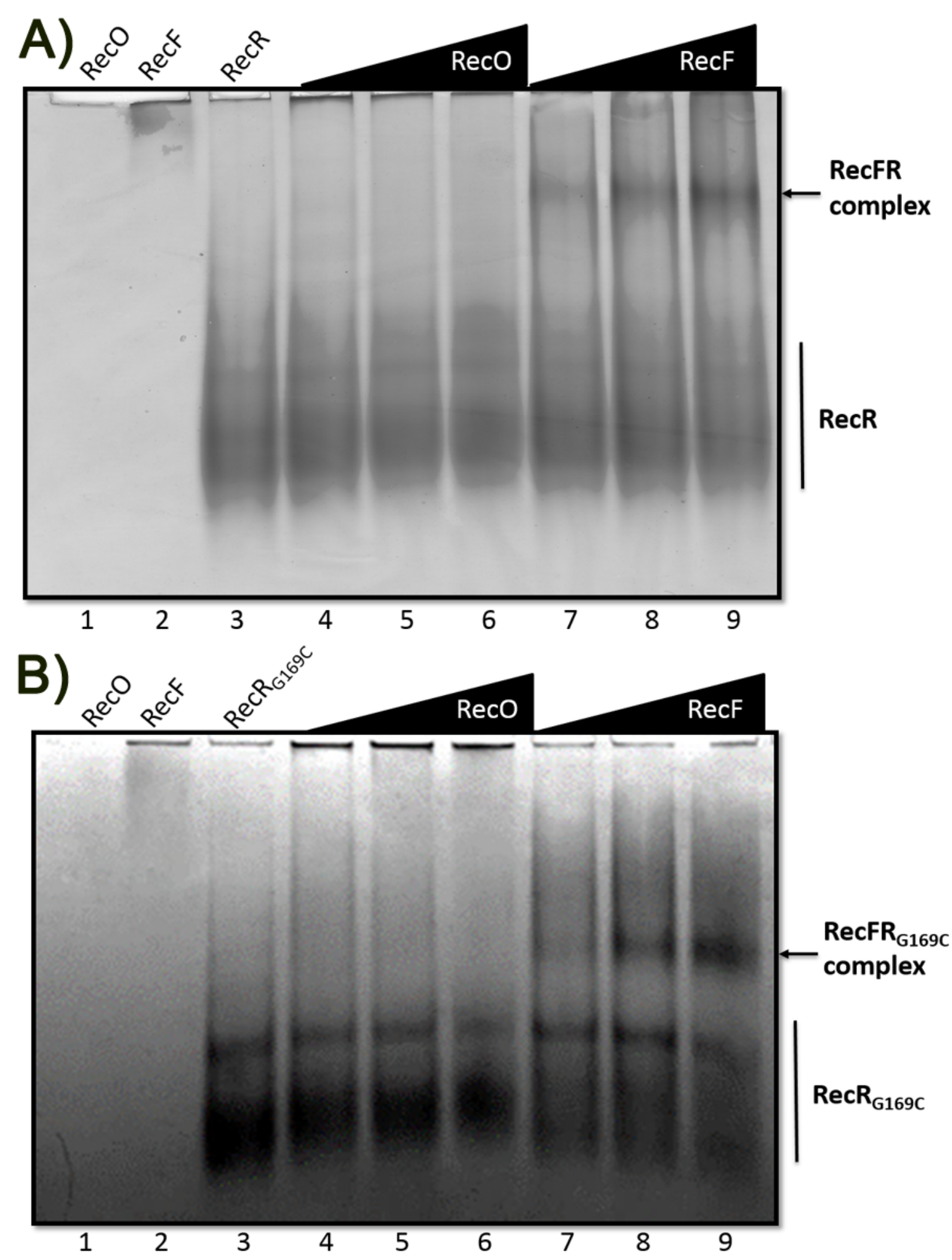


\section{Figure 7.}

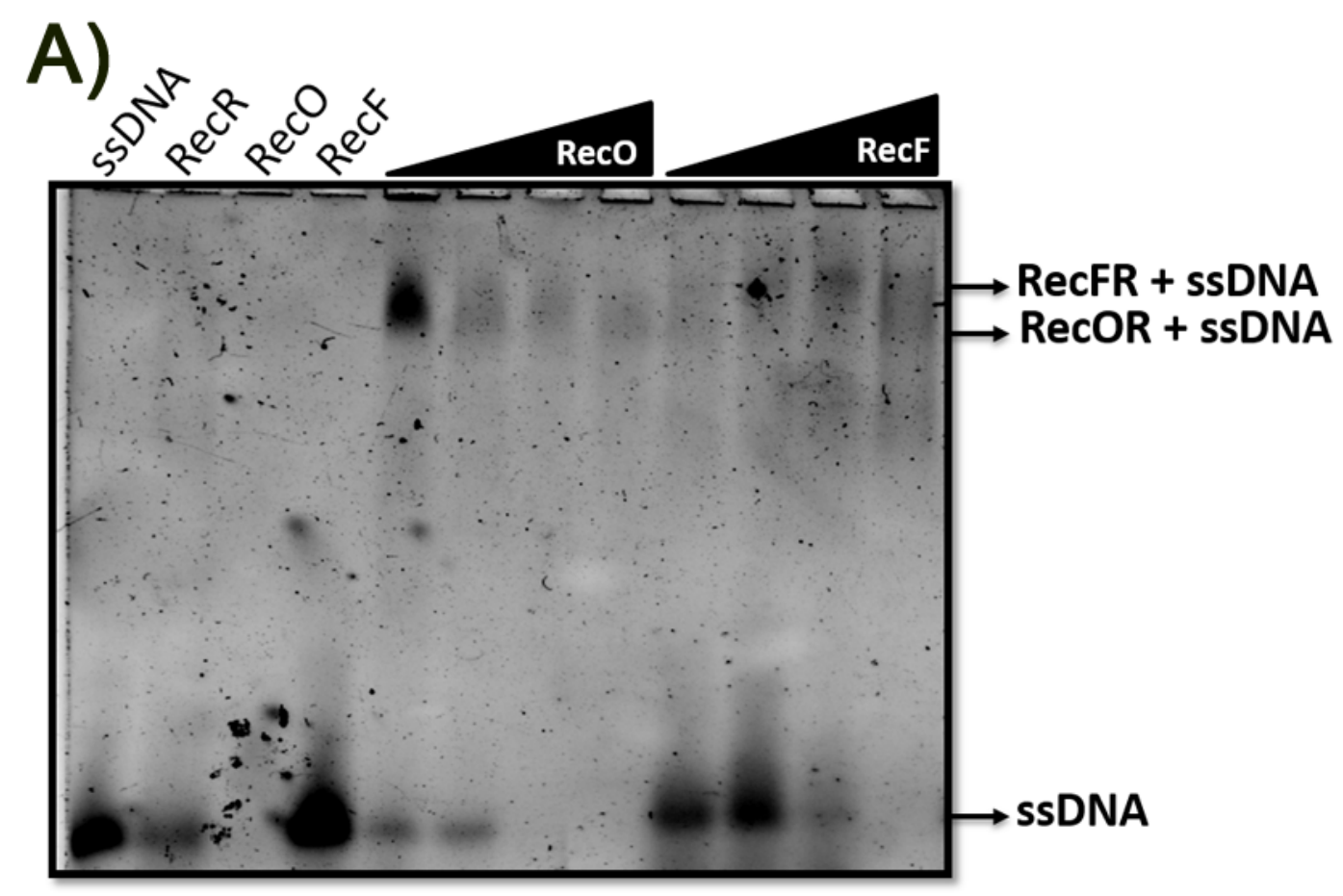

$\begin{array}{llllllllllll}1 & 2 & 3 & 4 & 5 & 6 & 7 & 8 & 9 & 10 & 11 & 12\end{array}$

B)
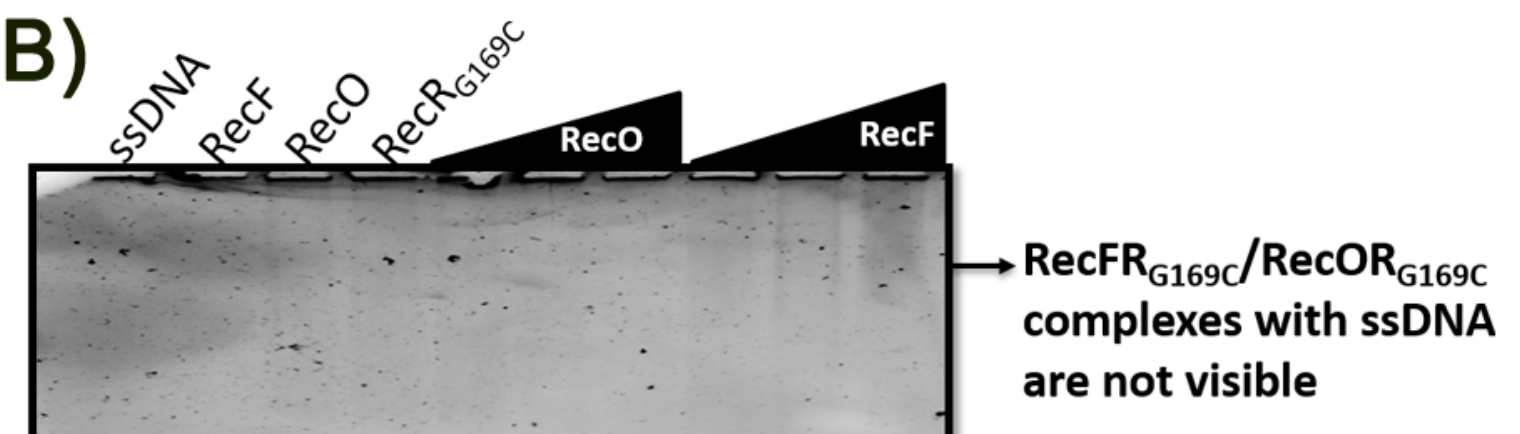

c

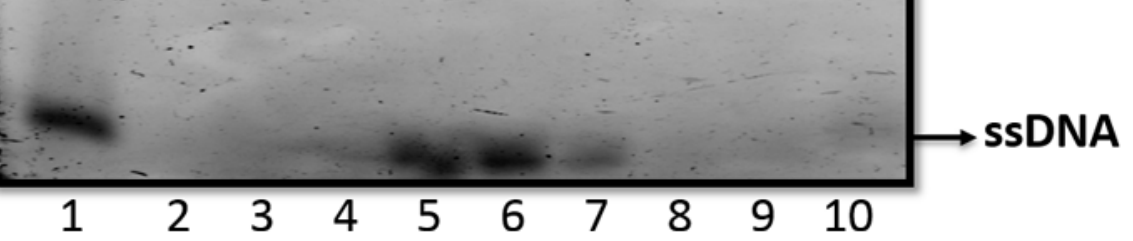


Figure 8.

(A)

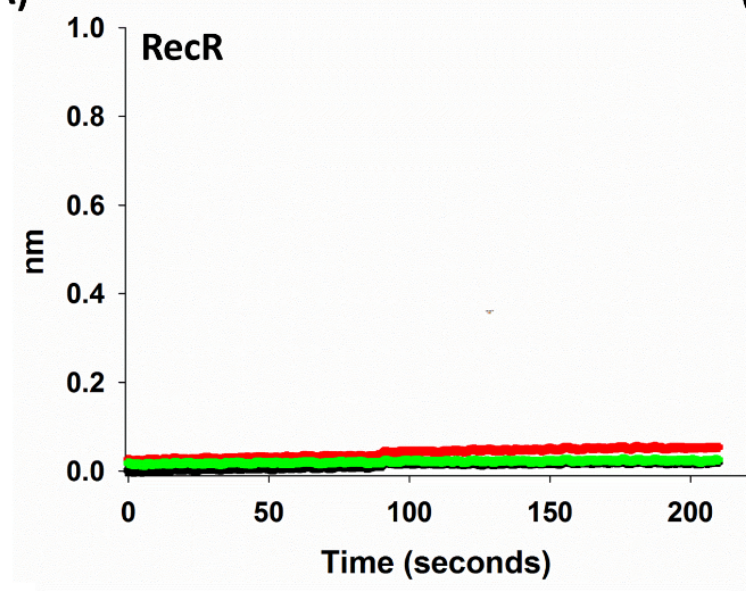

(C)

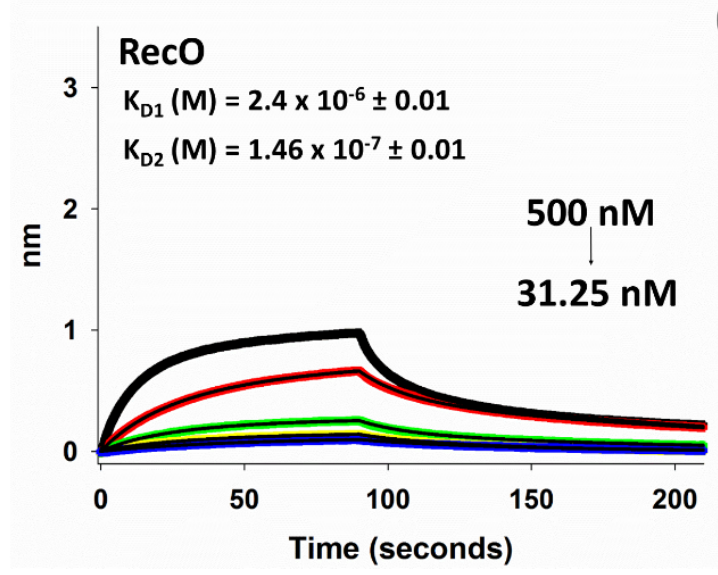

(B)

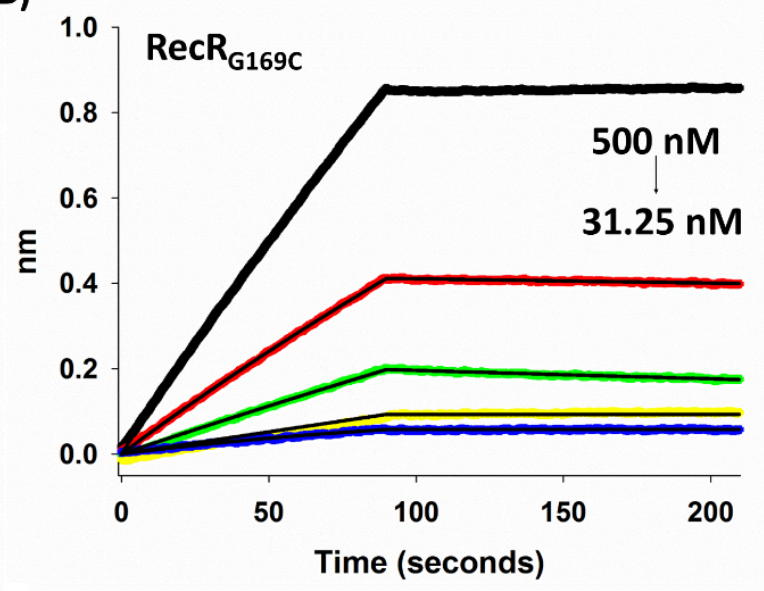

(D)

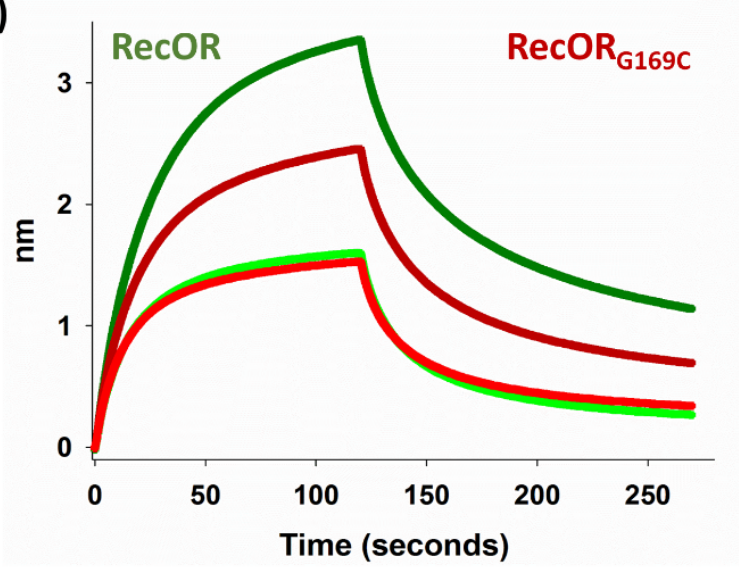


Figure 9.

(A)

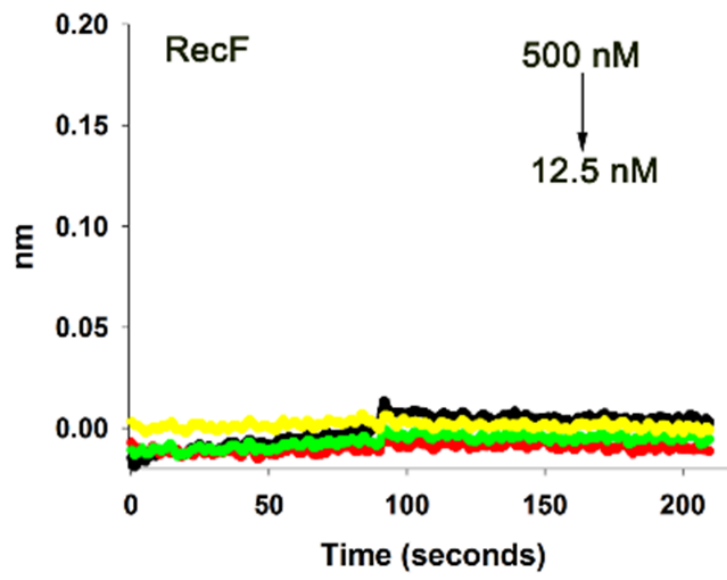

(B)

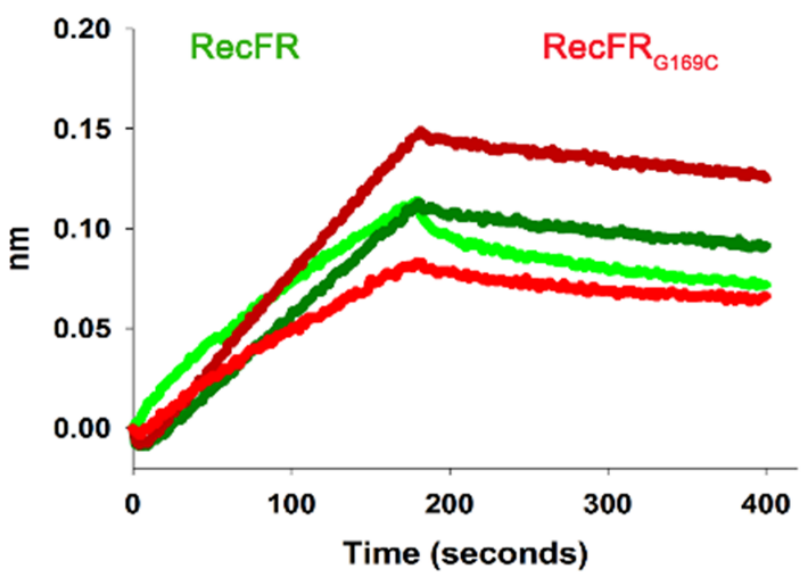

Figure 10.

(A)

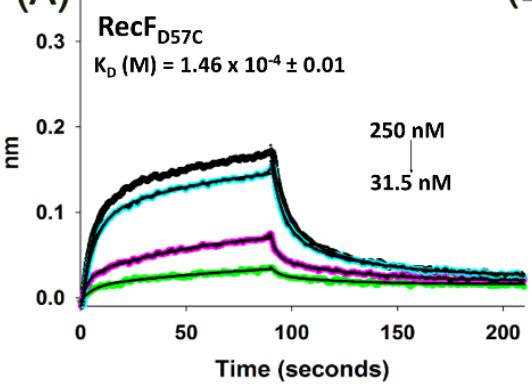

(B)

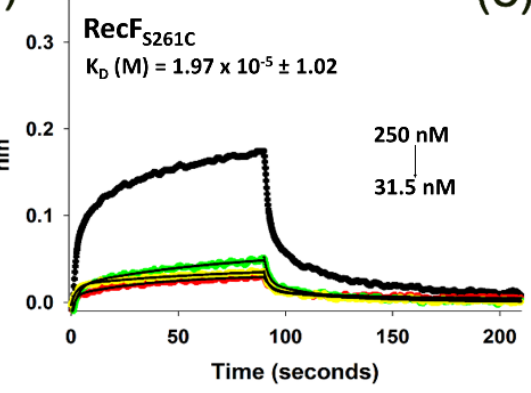

(C)

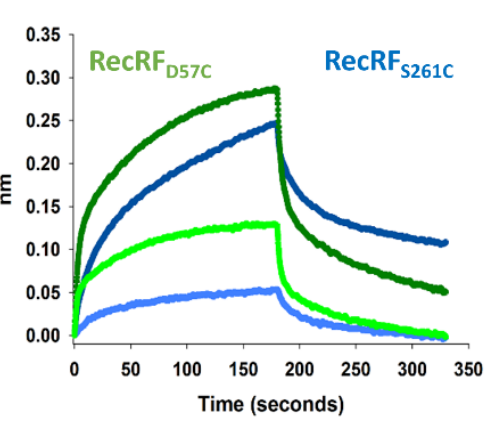


Figure 11.

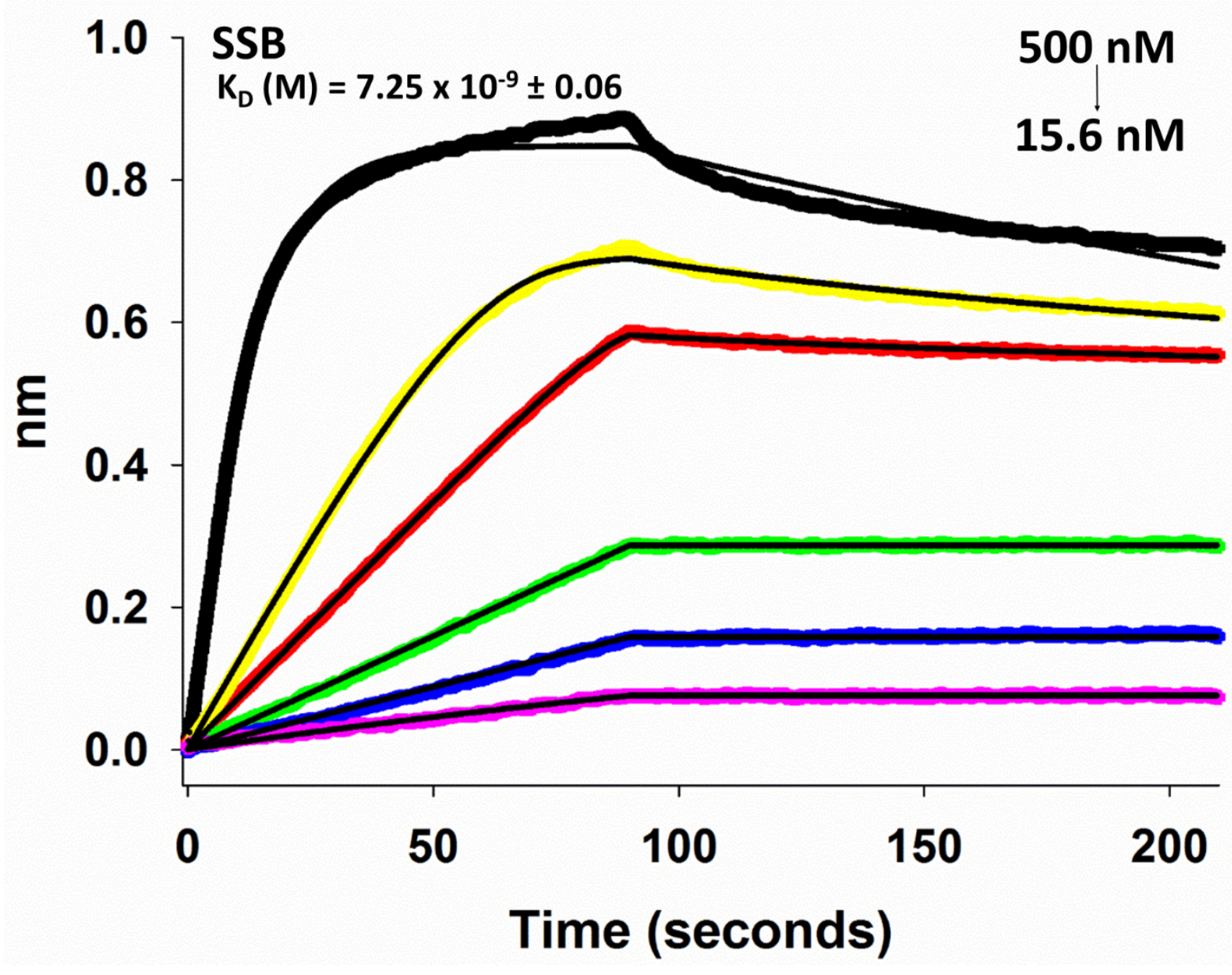


Figure 12.

(A)

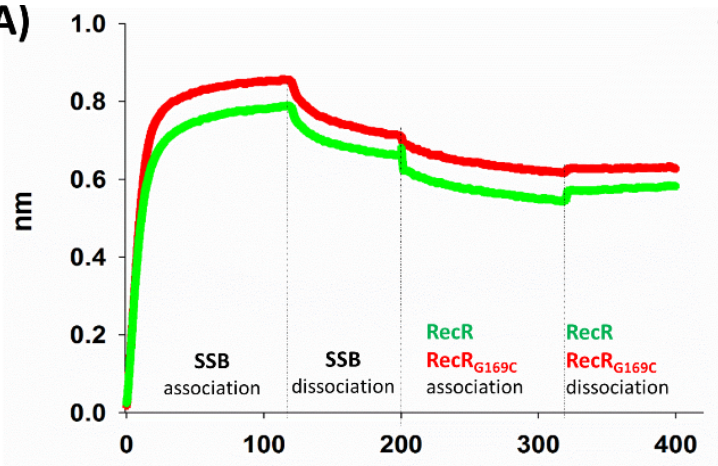

(C)

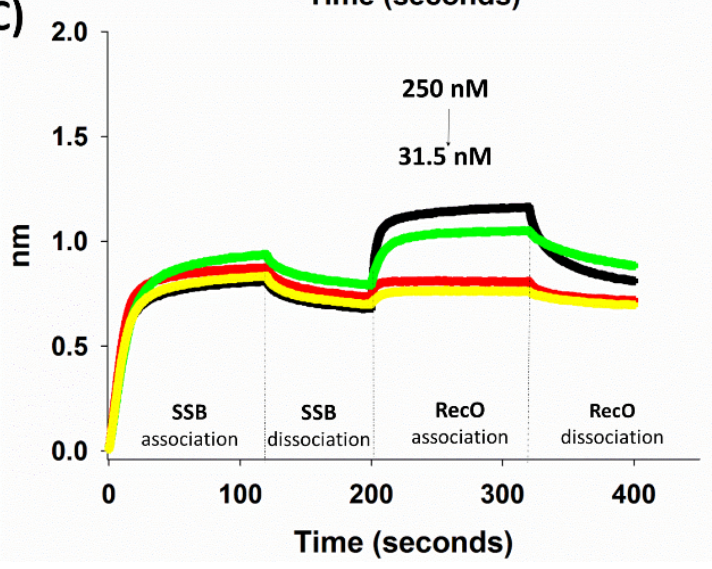

(B)

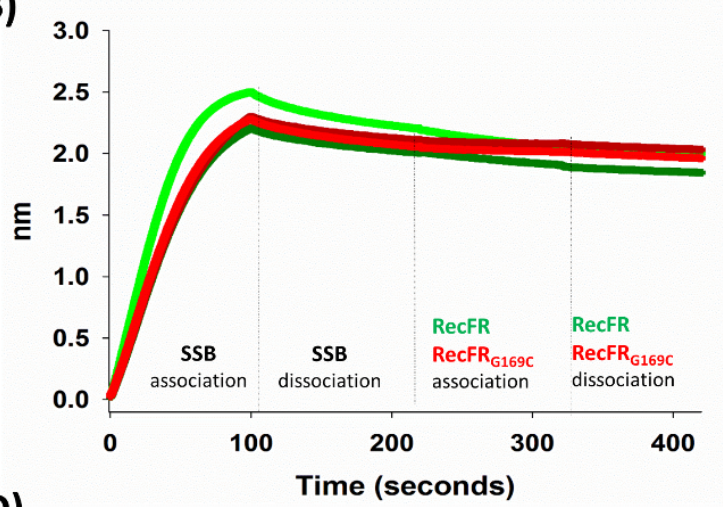

(D)

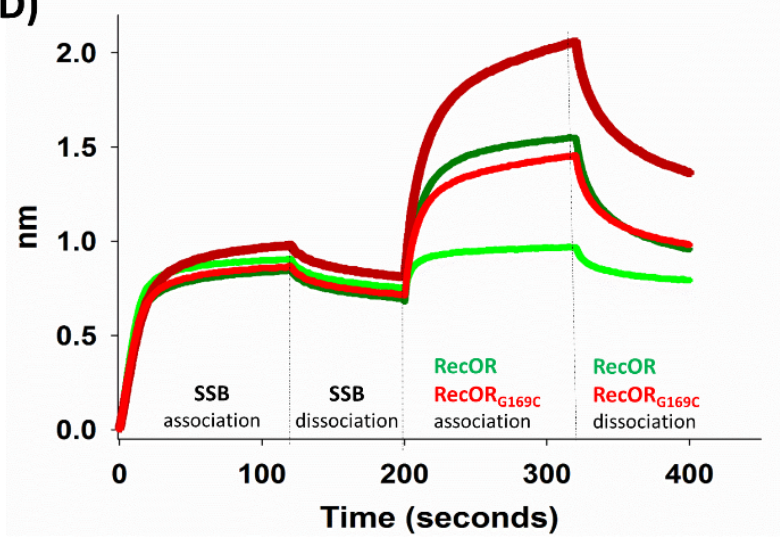




\section{Supplementary Information}

\section{Roles of oligomeric states of RecFOR pathway proteins in protein-protein and protein-}

\section{DNA interactions}

Santosh Kumar Chaudhary ${ }^{\mathrm{a}}$, Mohanapriya Elayappan ${ }^{\mathrm{b}}$, Jeyaraman Jeyakanthan ${ }^{\mathrm{c}}$ and Kanagaraj Sekar ${ }^{\mathrm{b}}$

${ }^{a}$ Department of Physics, Indian Institute of Science, Bangalore 560012, India

${ }^{b}$ Department of Computational and Data Sciences, Indian Institute of Science, Bangalore 560012, India

${ }^{\mathrm{c}}$ Department of Bioinformatics, Alagappa University, Karaikudi 630003, India

\section{Figures}

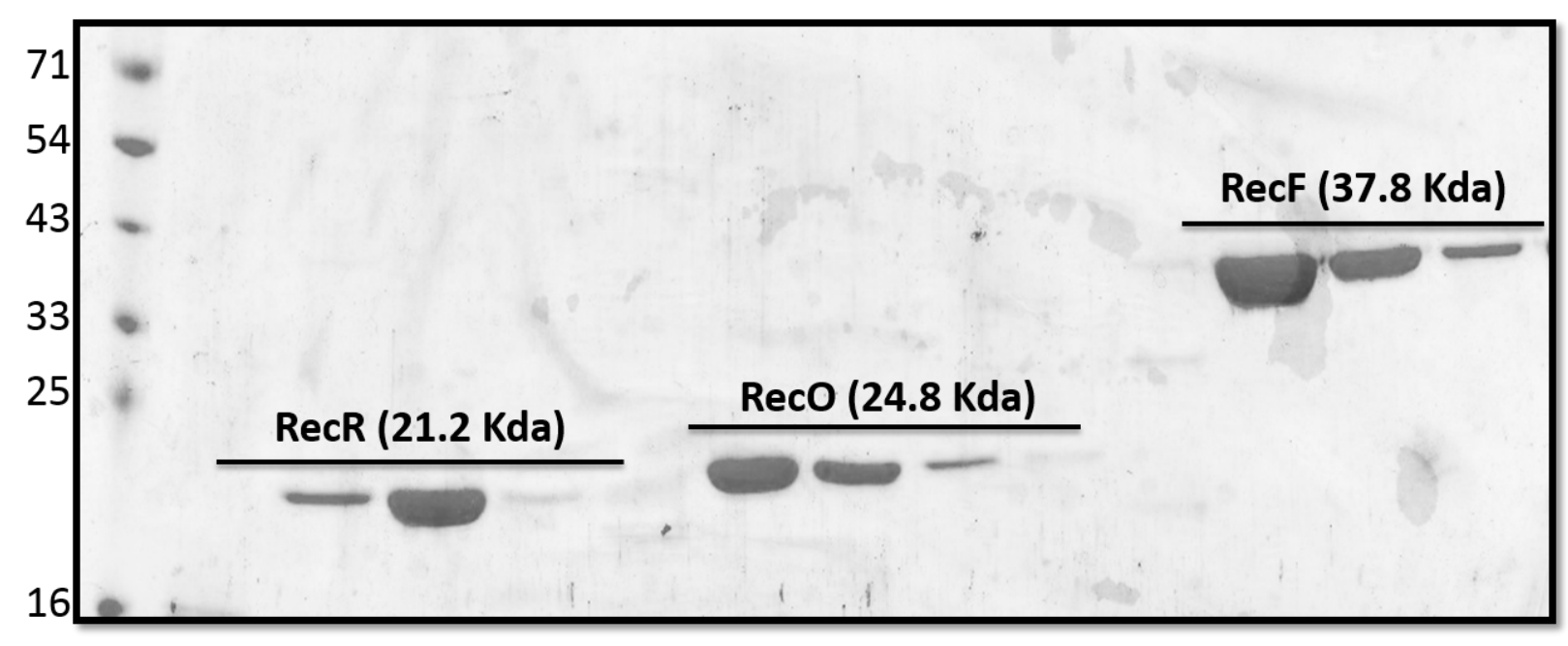

Figure S1. SDS-PAGE analysis of the purified TtRecR, TtRecO and TtRecF proteins. 

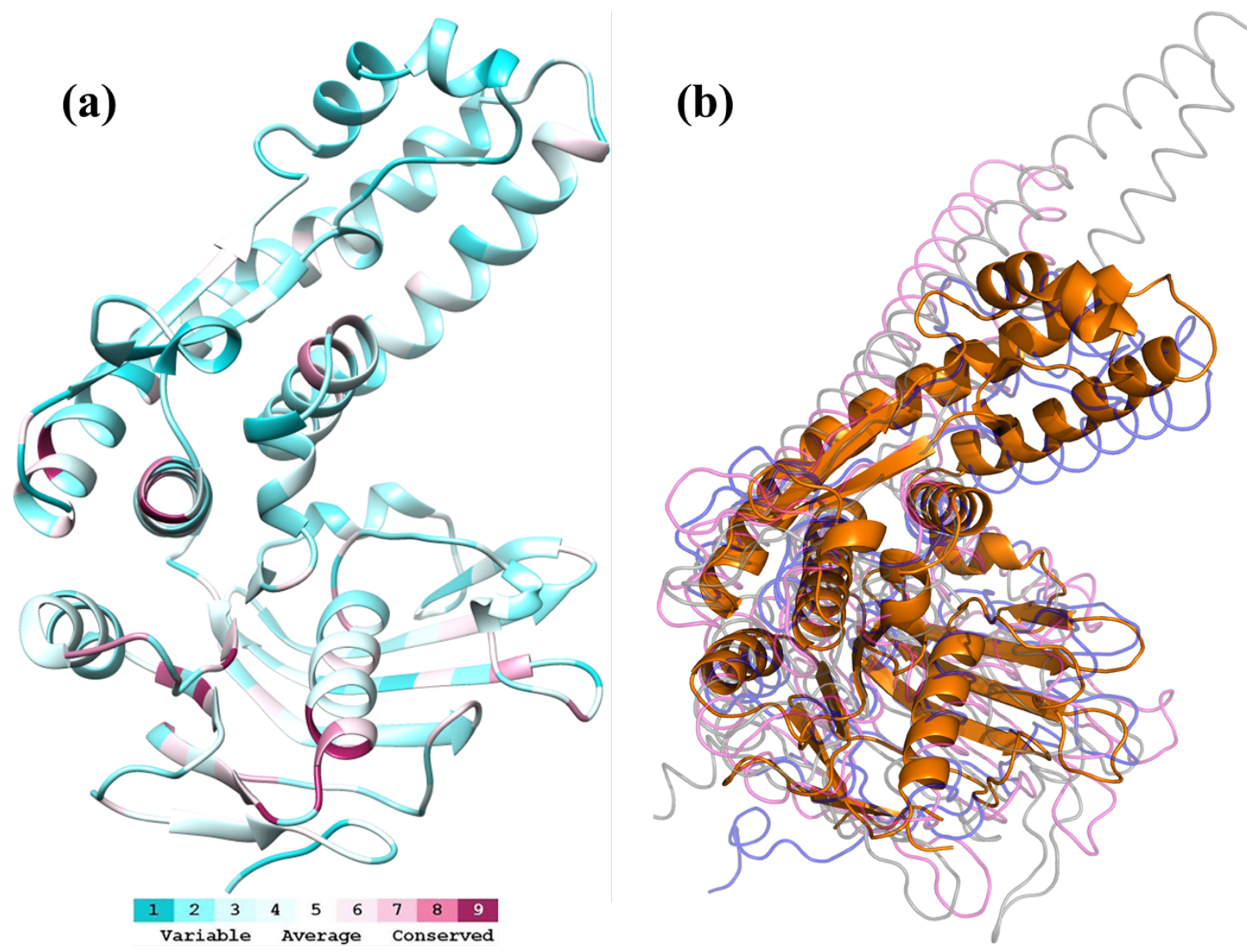

Figure S2. Sequence conservation and superposition of ttRecF protein with Rad50 and SMC proteins. (a) The ttRecF structure is colored based on the conservation score obtained from the alignment of ttRecF with drRecF, Rad50 (Pyrococcus furiosus) and SMC proteins (Pyrococcus furiosus). (b) Superposition of TtRecF with DrRecF, Rad50 (Pyrococcus furiosus) and SMC proteins (Pyrococcus furiosus). 
(a)

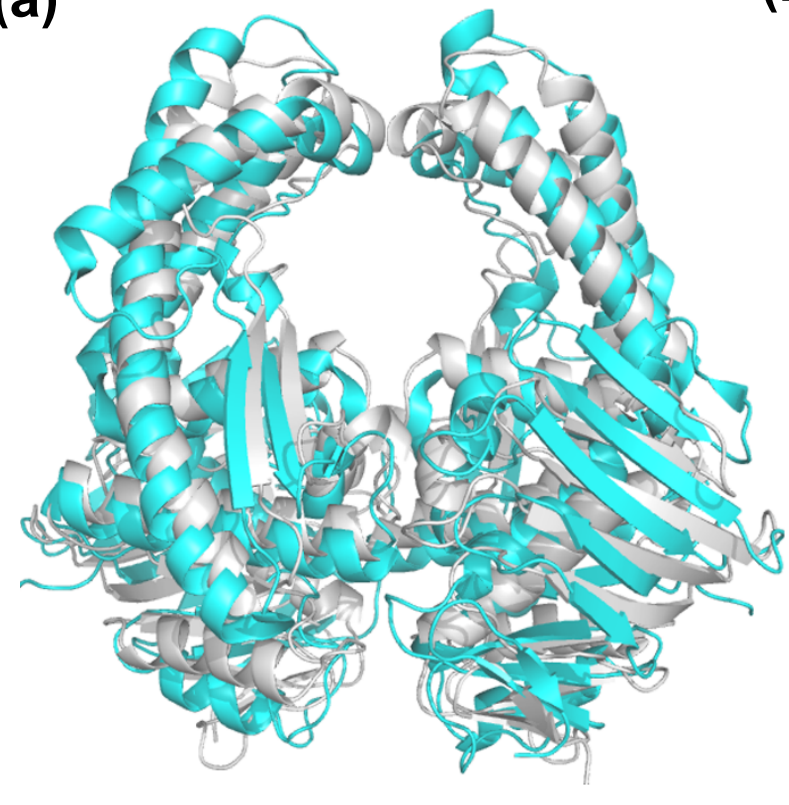

(b)

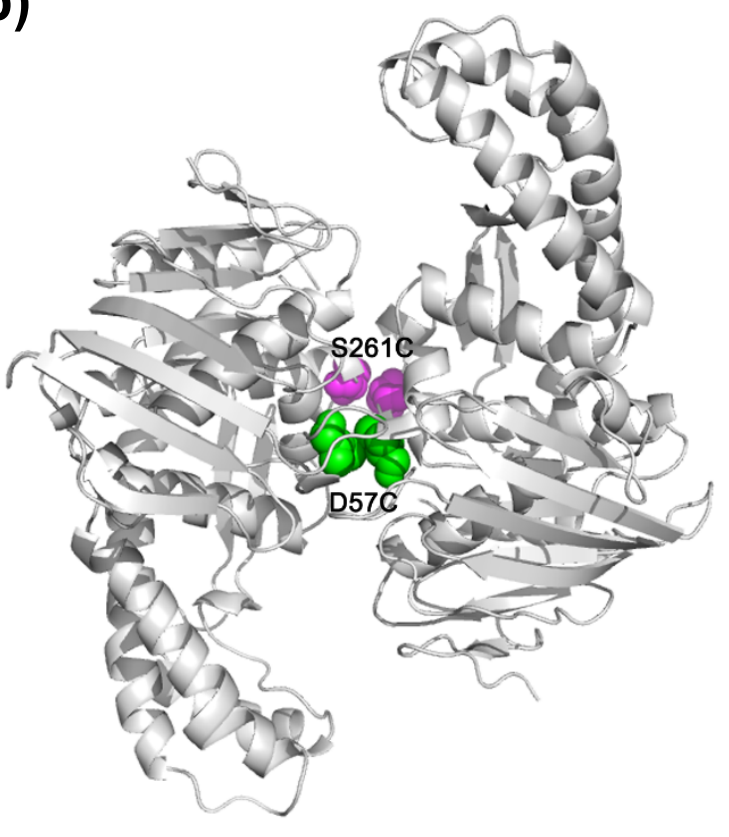

Figure S3. (a) Alignment of the dimeric TteRecF with the modelled dimeric TtRecF (b) The possible orientation of the mutant dimeric $\mathrm{TtRecF}_{\mathrm{S261}} / \mathrm{TtRecF}_{\mathrm{D57C}}$ proteins. 


\section{Tables}

Table S1. Primer sequences used for the mutagenesis in TtRecF and TtRecR proteins.

\begin{tabular}{|l|l|}
\hline Primer Name & Primer Sequence \\
\hline RF_S261C_Fwd & TGCCGCGGGGAGGCCAAGAC \\
\hline RF_S261C_rev & GGCGAACCGGTGGGCGGGC \\
\hline RF_D57C_Fwd & TGCCTCGTCCGCTTCGGGGAG \\
\hline RF_D57C_Rev & GGCAAGGCCCAGGGGGACCT \\
\hline RR_G169C_Fwd & TGCCTCCCCGTGGGGGGGAG \\
\hline RR_G169C_Rev & GTAGGCGGGCCGGGTCACCC \\
\hline
\end{tabular}


Table S2. Interface comparison between the dimeric TteRecF and the modelled dimeric TtRecF.

\begin{tabular}{|l|l|l|}
\hline Title & TteRecF & TtRecF \\
\hline Buried area upon the complex formation $(\AA)$ & 1347.0 & 2310.5 \\
\hline Buried area upon the complex formation $(\%)$ & 3.84 & 6.77 \\
\hline Interface area $(\AA)$ & 673.5 & 1155.25 \\
\hline Interface area Chain A (\%) & 3.83 & 6.78 \\
\hline Interface area Chain B (\%) & 3.85 & 6.77 \\
\hline $\begin{array}{l}\text { POLAR Buried area upon the complex } \\
\text { formation }(\AA)\end{array}$ & 786.0 & 1265.5 \\
\hline $\begin{array}{l}\text { POLAR Buried area upon the complex } \\
\text { formation }(\%)\end{array}$ & 58.35 & 54.77 \\
\hline POLAR Interface area $(\AA)$ & 393.0 & 632.75 \\
\hline $\begin{array}{l}\text { NO POLAR Buried area upon the complex } \\
\text { formation }(\AA ⿻)\end{array}$ & 561.0 & 1045.1 \\
\hline $\begin{array}{l}\text { NO POLAR Buried area upon the complex } \\
\text { formation }(\%)\end{array}$ & 41.65 & 45.23 \\
\hline NO POLAR Interface area $(\AA)$ & 280.5 & 522.55 \\
\hline$\#$ residues at the interface & 49 & 78 \\
\hline Residues at the interface of Chain A & 25 & 38 \\
\hline Residues at the interface of Chain B & 24 & 40 \\
\hline
\end{tabular}

